\title{
An efficient, selective collisional ejection mechanism for inner-shell population inversion in laser-driven plasmas
}

\author{
W. Andreas Schroeder, T.R. Nelson ${ }^{\dagger}$, A.B. Borisov, J.W. Longworth, K. Boyer, \\ and C.K. Rhodes \\ Department of Physics (M/C 273), University of Illinois at Chicago, \\ 845 W. Taylor Street, Chicago, IL 60607-7059, USA

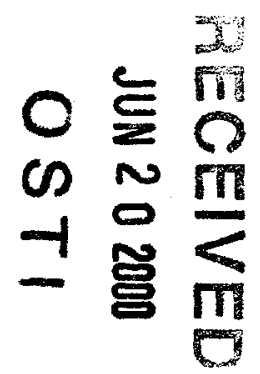

\section{Abstract}

A theoretical analysis of laser-driven collisional ejection of inner-shell electrons is presented to explain the previously observed anomalous kilovolt L-shell $x$-ray emission spectra from atomic Xe cluster targets excited by intense sub-picosecond $248 \mathrm{~nm}$ ultraviolet radiation [A. McPherson et al., Nature 370, pp. 631-634 (1994)]. For incident ponderomotively-driven electrons photoionized by strong above threshold ionization, the collisional ejection mechanism is shown to be highly $l$-state and significantly $n$-state (i.e. radially) selective for time periods shorter than the collisional dephasing time of the photoionized electronic wavefunction. The resulting preference for the collisional ejection of $2 p$ electrons by an ionized $4 p$ state produces the measured anomalous $\mathrm{Xe}(\mathrm{L})$ emission which contains direct evidence for (i) the generation of $\mathrm{Xe}^{27+}\left(2 p^{5} 3 d^{10}\right)$ and $\mathrm{Xe}^{28+}\left(2 p^{5} 3 d^{9}\right)$ ions exhibiting inner-shell population inversion and (ii) a coherent correlated electron state collision responsible for the production of double $2 p$ vacancies. For longer time periods, the selectivity of this coherent impact ionization mechanism is rapidly reduced by the combined effects of intrinsic quantum mechanical spreading and dephasing - in agreement with the experimentally observed and extremely strong $\sim \lambda^{-6}$ pump-laser wavelength dependence of the efficiency of inner-shell $(2 p)$ vacancy production in Xe clusters excited in underdense plasmas [K. Kondo et al., J. Phys. B 30, pp. 2707-2716 (1997)].

${ }^{\dagger}$ Present address: Sandia National Laboratories, MS-1188, P.O. Box 5800, Albuquerque, NM 87185-1188

Tel:

(312) $996-2974$

FAX: - (312) 996-9016

E-mail: andreas@uic.edu 


\section{DISCLAIMER}

This report was prepared as an account of work sponsored by an agency of the United States Government. Neither the United States Government nor any agency thereof, nor any of their employees, make any warranty, express or implied, or assumes any legal liability or responsibility for the accuracy, completeness, or usefulness of any information, apparatus, product, or process disclosed, or represents that its use would not infringe privately owned rights. Reference herein to any specific commercial product, process, or service by trade name, trademark, manufacturer, or otherwise does not necessarily constitute or imply its endorsement, recommendation, or favoring by the United States Government or any agency thereof. The views and opinions of authors expressed herein do not necessarily state or reflect those of the United States Government or any agency thereof. 


\section{DISCLAIMER}

Portions of this document may be illegible in electronic image products. Images are produced from the best available original document. 


\section{Introduction}

Several methods have been previously evaluated in attempts to generate coherent radiation in the extreme ultraviolet (XUV) and $\mathrm{x}$-ray spectral regions. Most notable amongst these are: highorder harmonic generation [1-3]; discharge-pumped soft $x$-ray lasers [4]; laser-pumped recombination [5,6], electron-impact ionization [7,8], and hybrid collision-recombination [9] sources; $\mathrm{x}$-ray [10] and ponderomotively-generated Larmor radiation [11] excited emitters; and Thomson scattering sources [12]. However, none of these methods have been successful at generating coherent radiation with a brightness in excess of $10^{30} \gamma \mathrm{s}^{-1}(\mathrm{mrad})^{-2}(\mathrm{~mm})^{-2}(0.1 \% \mathrm{BW})^{-1}$ at a wavelength $\lambda<10 \AA$; that is, a kilovolt $\mathrm{x}$-ray brightness level sufficient for interferometric biological imaging capable of providing an atomic-level resolution visualization of the molecular anatomy of cells, tissues and organisms in the natural state [13]. Indeed, to date, kilovolt $\mathrm{x}$-ray imaging applications have been limited to static [14] and dynamic [15] differential absorption measurements and time-resolved diffraction experiments [16] using incoherent radiation from laser-driven plasmas. Moreover, practical limitations in the generation of high-power sub-5-fs laser pulses and the spectral merging of harmonic orders above $\sim 200$ may preclude the development of a high brightness source of $>1 \mathrm{keV}$ photons by high harmonic generation techniques, while a recent analysis of linear and nonlinear (i.e. multiple) electron-photon interactions [17] suggests that Thomson scattering sources may never reach a brightness level sufficient for a ground-breaking interrogation of living organisms.

A potentially very effective method of producing an extremely bright source of kilovolt $\mathrm{x}$-ray radiation would be to generate a controlled population inversion in the inner shells of high- $Z$ atoms, i.e. produce specific species of "hollow atoms" [18]. In this paper, we outline a mechanism for achieving precisely this type of population inversion in laser-driven plasmas. The mechanism is based on the selective collisional ionization of inner-shell electrons by ponderomotively-driven electrons initially photoionized by strong above threshold ionization (ATI) [19]. The selectivity relies on the fact that the wavefunction of a photoionized electron distribution maintains its phase and geometric symmetry for a time shorter than the charateristic collisional dephasing time which, by comparison with electron-electron scattering rates in semiconductors $[20,21]$, is of the order of one femtosecond $\left(10^{-15} \mathbf{s}\right)$. Consequently, if the photoionized wavefunction collides with an ion or atom within this time period, the matrix element associated with the collision will be dominated by the intrinsic dynamics of the quantum mechanical spreading of the photoionized atomic orbital. In stark contrast to a "plane-wave" collision, this results in a strong angular momentum ( $l$ - and $m$-state) and a significant radial $(n$ state) dependence in the selectivity of the interaction. Laser-driven classically free electrons in the 
plasma [9] cannot exhibit this selectivity, since their wavefunctions contain no information about the atomic orbitals from which they were ionized.

The essential features of the selective collisional ionization mechanism will be discussed in Section III using the interaction of ponderomotively-driven $4 p$ electrons photoionized from Xe with the bound state electrons of Xe ions as an example. The results of this theoretical analysis are shown to be in good agreement with the observed anomalous $\mathrm{Xe}(\mathrm{L})$ spectra described in Section II, which were obtained under intense ultraviolet (UV) $248 \mathrm{~nm}$ laser irradiation of 5-20 atom Xe cluster targets [22,23]. Specifically, it explains the strongly prefered generation of $2 p$ vacancies in Xe cluster targets; a process leading naturally to the observed evidence for $(i)$ a laser-driven generation of Xe ions exhibiting population inversion between the $2 p$ and $3 d$ shells and (ii) the production of multiple $2 p$ vacancies [18]. Furthermore, for gaseous targets such as Xe clusters, the mechanism displays a substantial pump-laser wavelength scaling favoring the use of shorter wavelength driving sources - in agreement with the experimental determination of at least a $\lambda^{-6}$ scaling in the efficiency of Xe L-shell (2.5-3.1 $\AA$ ) emission between $248 \mathrm{~nm}$ and $800 \mathrm{~nm}$ laser excitation $[24,25]$. The possible extension of this technique to the selective generation of $1 \mathrm{~s}$ vacancies in $\mathrm{Kr}$ for strong $\sim 1 \AA \mathrm{K}$-shell emission is explored in Section IV.

\section{Xe L-Shell Emission}

Arguably the strongest experimental evidence supporting the existence of an anomalous selective laser-driven collisional ionization mechanism comes from the L-shell $(3 d \rightarrow 2 p)$ hollow-atom emission observed from atomic Xe clusters [18] under intense irradiation $\left(10^{18}-10^{19} \mathrm{~W} / \mathrm{cm}^{2}\right)$ by sub-picosecond ultraviolet (UV) terawatt $\left(1 \mathrm{TW}=10^{12} \mathrm{~W}\right)$ laser pulses at $248 \mathrm{~nm}$ generated by a hybrid Ti:Sapphire/KrF* laser system [26]. As described elsewhere [18,23,25,27-29], these TW UV laser pulses are focused by an $f / 3$ optic into a target of Xe clusters (typically of 5-20 atoms) generated by a cooled pulsed valve. A mica-crystal von Hámos spectograph equipped with Kodak RAR 2492 x-ray film and appropriate filters was used to collect the spectrum of the $x$-ray radiation emitted by the irradiated target. As shown in Figure 1, the kilovolt Xe L-shell spectra detected under these experimental conditions displays the characteristic double peaked hollowatom structure of the $3 d \rightarrow 2 p$ transition [30]. It is important to note that this $\mathrm{Xe}(\mathrm{L})$ emission must be generated by a collisional mechanism in which photoionized electrons are accelerated by the laser field to a sufficient energy to eject an inner-shell $2 p$ electron via Coulombic interaction. This is because the Xe $2 p$ state, which has an ionization potential of $\sim 8 \mathrm{keV}$, cannot be directly photoionized by above threshold ionization (ATI) [19] at irradiances below $10^{22} \mathrm{~W} / \mathrm{cm}^{2}$. 
However, a fully relativistic classical model of the photoionized electron trajectories for $248 \mathrm{~nm}$ irradiation at $10^{18}-10^{19} \mathrm{~W} / \mathrm{cm}^{2}$ [25] has shown that the ponderomotively-driven electrons ionized from more weakly bound orbitals can return to their cluster of origin with an energy of $10-20 \mathrm{keV}$; a value sufficient to eject $2 p$ electrons collisionally and thus produce the observed L-shell emission.

A striking feature of the spectra shown in Fig. 1 is the strong L-shell emission due to single $2 p$ shell vacancies from $\mathrm{Xe}^{+q}$ ions for charge states of $q=27$ to 37 corresponding to $2 p \leftarrow 3 d$ transitions ranging from a filled $3 d$-shell $\left(2 p^{6} 3 d^{9} \leftarrow 2 p^{5} 3 d^{10}\right)$ to one containing only one electron $\left(2 p^{6} \leftarrow 2 p^{5} 3 d\right)$ respectively. This observation is very unusual in light of the magnitudes of the cross-sections evaluated from a single-electron plane-wave analysis for the collisional ejection of inner-shell electrons with an ionization potential $I_{\mathrm{p}}>250 \mathrm{eV}$ [31,32]. As shown in Figure 2, a plane-wave collisional analysis indicates that an electron incident on a Xe ion with a collisional energy $E_{\text {coll. }}$ of $10-20 \mathrm{keV}$ is $\sim 10$ times more likely to eject a $3 d, 3 p$, or $3 s$ electron with ionization potentials of $\sim 2-3 \mathrm{keV}$ than a $2 p$ electron with $I_{\mathrm{p}} \approx 8 \mathrm{keV}$. This analysis is clearly in stark contradiction to the experimental observations of Fig. 1 on two grounds. First, it would predict that $\mathrm{Xe}(\mathrm{L})$ emission from $\mathrm{Xe}^{+q}$ ions should be approximately an order of magnitude less than that from $\mathrm{Xe}^{+}(q+1)$ ions, ultimately suggesting that on average all $3 d$ electrons should suffer collisional ejection before a single $2 p$ electron is ejected. Consequently, the experimental observation of significant $\mathrm{L}$-shell emission from Xe $\mathrm{X}^{27+}$ with a filled $3 d$ shell (i.e. a $2 p^{6} 3 d^{9} \leftarrow 2 p^{5} 3 d^{10}$ transition) indicates that the collisional cross-section for ejection of a $2 p$ electron could be at least an order of magnitude larger than that for the ejection of a $3 d$ electron under $248 \mathrm{~nm}$ UV irradiation at $10^{18}-10^{19} \mathrm{~W} / \mathrm{cm}^{2}$; that is, precisely the inverse of the scaling associated with the plane-wave collisional analysis. And second, it suggests that a significant number of $3 p$ and $3 s$ vacancies should also be generated by the collisional mechanism ejecting the $2 p$ electrons. Yet, strong emission from ionic species possessing such vacancies has not been observed.

More anomalous, given the plane-wave collisional analysis, is the observation of spectral features associated with double $2 p$ vacancies in the range $2.60-2.65 \AA$ (Fig. 1) $[18,22,23]$. This result is even more astonishing when one considers the fact that the lifetime of a single $2 p$ vacancy is only $\sim 1.8 \mathrm{fs}[32,30]$. This means that the generation of $2 p$ vacancies in the collisional ejection mechanism must be occuring at a rate in excess of $\sim 10^{15} \mathrm{~s}^{-1}$; that is, effectively producing multiple $2 p$ vacancies within one optical cycle of the $248 \mathrm{~nm}$ pump-laser radiation used in the experiment. Previous analyses $[28,33,34]$ have suggested that this could be accomplished by ordered or cooperative ponderomotive electronic motions in which $Z$ photoionized electrons behave as a single quasi-particle of charge $Z e$ with an effective collective energy $Z$ times their individual mean 
energy. For example, the cooperative action of 3-4 electrons under $248 \mathrm{~nm}$ UV irradiation at $10^{18}-10^{19} \mathrm{~W} / \mathrm{cm}^{2}$ would certainly enhance the probability of collisional $2 p$ vacancy generation by ensuring that the quasi-particle has an effective collisional energy of $\sim 40 \mathrm{keV}$, i.e. near the peak of the $2 p$ cross-section (Fig. 2). However, such a mechanism fails to explain the apparent selective generation of $2 p$ vacancies with a rate exceeding the production of other multiple inner-shell vacancies (e.g. $2 s, 3 s$, and $3 p$ vacancies) in the Xe ions.

It is also interesting to note that a comparison experiment conducted with a TW femtosecond Ti:sapphire laser system operating in the infrared (IR) at $800 \mathrm{~nm}$ did not reveal the generation of multiple $2 p$ vacancies in the observed Xe L-shell emission $[24,25]$. Moreover, the overall strength of the L-shell emission was observed to be three orders of magnitude $(-3000 \mathrm{x})$ weaker under IR irradiation. Consequently, any plausible collisional ionization mechanism must $(i)$ be selective in nature in order to explain the anomalous features of the $\mathrm{Xe}(\mathrm{L})$ emission outlined above under $\mathrm{UV}$ irradiation and (ii) be consistent with the experimental data obtained at both pump-laser wavelengths. Evidently, the latter effect could arise directly from a strong scaling with the laser excitation wavelength, specifically the pump-laser radiation period.

\section{Selective Collisional Ionization}

\section{III.A. Interaction Geometry and Matrix Element}

The cross-section for any collisional interaction is proportional to the modulus square of the matrix element $M_{f i}$ associated with the interaction mechanism [35] which, for an initial state $|i\rangle$ coupled to a final state $|f\rangle$ through the interaction potential $V$, is written as

$$
M_{f i}=\langle f|V| i\rangle .
$$

In our case, we are interested in the collisional ejection of bound inner-shell electrons by a ponderomotively-driven photoionized electron through the action of the Coulomb potential. The general interaction geometry for this collision is shown in Figure 3. The bound states $\left|\phi_{j}\right\rangle$ are defined by the radial coordinate $\mathbf{r}_{1}$ centered on the nucleus of the target atom or ion. The displacement vector $\mathbf{R}$ describes the trajectory of the center (centriod) of the photoionized electron distribution (or state) during the motion determined by the laser's oscillating ponderomotive potential and the Coulomb potential of the ionized atomic cluster $[25,36]$. The 
internal radial coordinate of the photoionized electron wavefunction $|\psi\rangle$ is given by $\mathbf{r}_{2}$ so that $\mathbf{R}+$ $\mathbf{r}_{2}$ defines the position of the ionized electron with respect to the target ion. Within this framework, the initial state $|i\rangle$ can be written as

$$
\langle\mathbf{r} \mid i\rangle=\phi_{j}\left(\mathbf{r}_{1}\right) \psi\left(\mathbf{r}_{2}, t\right) \exp \left[i \mathbf{k}_{0} .\left(\mathbf{R}+\mathbf{r}_{2}\right)\right]
$$

that is, as the product of the incident ponderomotively-driven time-dependent photoionized wavefunction $\left\langle\mathbf{R}+\mathbf{r}_{2} ; t \mid \psi\right\rangle$, for which $\mathbf{k}_{0}$ is the wavevector associated with its motion, and the stationary inner-shell electronic states of target atom or ion $\left|\phi_{j}\right\rangle$. In the limit of a sufficiently long time after the interaction, the final state $|f\rangle$ for the scattered and ejected inner-shell electrons may be well represented as the product of two spherical waves emanating from the origin of the coordinate system centered on the target atom (Fig. 3):

$$
\langle r \mid f\rangle=\frac{\exp \left[i \mathbf{k}_{1} \cdot \mathbf{r}_{\mathbf{l}}\right]}{r_{1}} \cdot \frac{\exp \left[\mathbf{i k}_{2} \cdot \mathbf{r}_{2}\right]}{r_{2}}
$$

Here, $\mathbf{k}_{1}$ and $\mathbf{k}_{2}$ are the wavevectors of the ejected and scattered incident electrons after the collision respectively. Thus, since the collisional ejection mechanism is subject the momentum conservation condition $k_{0}=k_{1}+k_{2}$ and the vector $r_{1}-\left(R+r_{2}\right)$ describes the displacement between the two electrons involved in the Coulomb interaction, the matrix element associated with the interaction is of the form

$$
M_{f i}=\iiint d^{3} \mathbf{r}_{1} d^{3} \mathbf{r}_{2} d \mathbf{R} \frac{\phi_{j}\left(\mathbf{r}_{1}\right) \psi\left(\mathbf{r}_{2}, t\right)}{r_{1} r_{2}\left|\mathbf{r}_{1}-\left(\mathbf{R}+\mathbf{r}_{2}\right)\right|} \exp \left[i \mathbf{k}_{0} \cdot \mathbf{R}-i \mathbf{k}_{1} \cdot\left(\mathbf{r}_{1}-\mathbf{r}_{2}\right)\right]
$$

In general, the full exact calculation of this matrix element is highly complex [37,38]. Accordingly, for the scope of this paper, we will develop a suitable approximate analysis based upon the fact that the $1 / \mathbf{r}$ dependence of the Coulomb potential will strongly select collisional interactions between $|\psi\rangle$ and $\left|\phi_{j}\right\rangle$ for which $\mathbf{R} \approx 0$, i.e. interactions for which the center of the photoionized state $|\psi\rangle$ passes close to the nucleus of the target ion. Consequently, the essential physics behind the cross-section for inner-shell collisional ejection is expected to be obtained from an evaluation of $\left|M_{f i}(\mathbf{R}=0)\right|^{2}$. In particular and notwithstanding this approximation, it is immediately evident from equation (4) that the strength of the matrix element will be strongly dependent upon the overlap between $|\psi\rangle$ and $\left|\phi_{j}\right\rangle$; that is, the form of $|\psi\rangle$ since the bound states $\left|\phi_{j}\right\rangle$ are known. Specifically, the spherical symmetry of the incident photoionized wavefunction 
$|\psi\rangle$ with respect to the target wavefunctions $\left|\phi_{j}\right\rangle$ should play a major role in determining the collisional cross-sections for particular inner-shell ejection mechanisms. Hence, as in atom-atom and ion-atom collisions [39], the interaction is expected to have strong geometrical features associated with the electronic charge distributions of $|\psi\rangle$ and $\left|\phi_{j}\right\rangle$.

\section{III.B. Temporo-Spatial Dynamics of Photoionized Electron Wavefunctions}

Before the collision of the electron with the target atom or ion, it must first be photoionized and then accelerated by the pump laser field. For laser irradiances above $\sim 10^{17} \mathrm{~W} / \mathrm{cm}^{2}$, such as those of $10^{18}-10^{19} \mathrm{~W} / \mathrm{cm}^{2}$ required to generate the observed $\mathrm{Xe}(\mathrm{L})$ emission (Fig. 1), above threshold ionization (ATI) is the dominant ionization process [19]. Moreover, under our experimental conditions, the incident UV pump-laser radiation is observed to undergo relativistic self-focusing, resulting in channelled beam propagation over more than 100 Rayleigh ranges [40]. Numerical simulations of the self-focusing mechanism have indicated that significant self-steepening of the leading edge of the laser pulse occurs over irradiances of $\sim 10^{17}-10^{22} \mathrm{~W} / \mathrm{cm}^{2}$ as it propagates down the channel [41]. As a result, the conditions of photoionization experienced by the irradiated atoms or ions are expected to be highly dynamic. In particular, it is very probable that the photoionization mechanism closely follows the "ionization ignition" model of Rose-Petruck et al. [42] where entire orbital shells may be ionized by ATI at the peak laser field in each half cycle of the radiation. Thus, for our experimental irradiances of $10^{18}-10^{19} \mathrm{~W} / \mathrm{cm}^{2}$, we may make the ansatz that the free electronic state is initially a replica of the photoionized bound state $\left|\phi_{j}\right\rangle$, i.e. the bound state is simply "tipped" out of the atomic or ionic potential by the perturbing ponderomotive potential of the strong laser field in $\sim 0.1 \mathrm{fs}$. Consequently, immediately after photoionization at $t=0$, we may write within this "sudden approximation" that

$$
\left\langle\mathbf{r}_{2} ; t=0 \mid \psi\right\rangle \approx\left\langle\mathbf{r}_{1} \mid \phi_{j}\right\rangle
$$

It follows that the initial photoionized electronic state $\left\langle\mathbf{r}_{2} ; t=0 \mid \psi\right\rangle$ possesses the angular and radial characteristics of $\left|\phi_{j}\right\rangle$, namely $R(r) Y_{l m}(\theta, \phi)$ where $R(r)$ and $Y_{l m}(\theta, \phi)$ are the usual radial eigenfunctions and spherical harmonics for a central Coulomb potential respectively. As a direct consequence, one would expect $\left\langle r_{2} ; t=0 \mid \psi\right\rangle$ to exhibit substantial $p$-wave symmetry within the irradiance range of $10^{18}-10^{19} \mathrm{~W} / \mathrm{cm}^{2}$ under which the $\mathrm{Xe}(\mathrm{L})$ emission of Fig. 1 was observed, since the six-electron Xe $4 p$ state with an ATI threshold of $\sim 2 \times 10^{18} \mathrm{~W} / \mathrm{cm}^{2}$ is one of the last states to be photoionized at these levels of irradiance. 
The initially ionized state $\left\langle\mathbf{r}_{2} ; t=0 \mid \psi\right\rangle$ is subsequently accelerated in the ponderomotive potential of the oscillating laser field $\mathbf{E} \cos \omega t[25,43]$. This process is usually described by the expansion of $|\psi\rangle$ in terms of Volkov states $\left|\phi_{\mu}\right\rangle[44]$, the eigenstates of the ponderomotive potential;

$$
|\psi\rangle=\sum_{\mu}\left|\phi_{\mu}\right\rangle\left\langle\phi_{\mu} \mid \psi\right\rangle
$$

However, the expansion coefficients $\left\langle\phi_{\mu} \mid \psi\right\rangle$ are generally not explicitly evaluated since, as we will demonstrate, the ionized electronic wavefunction evolves into phase-unrelated Volkov states $\left|\phi_{\mu}\right\rangle$ in a timescale significantly less than the 3-4fs radiation period of the most commonly used high-power IR laser systems [45]. The resulting loss of information on both the initial phase and symmetry of $|\psi\rangle$ does not allow any possible selectivity in collision process due to the details of the spatial overlap of the returning electron wavefunction $\left\langle\mathbf{r}_{2} ; t \mid \psi\right\rangle$ with the bound states $\left|\phi_{j}\right\rangle$ of the target atom or ion to be treated. Furthermore, to the authors' knowledge, the explicit time evolution of the photoionized state $|\psi\rangle$ due to intrinsic quantum mechanical spreading and collisional dephasing (due to internal scattering amongst the electrons in the ionized state $|\psi\rangle$ ) has not been previously considered. The evaluation of these effects and their affect on collisional ionization interactions is a crucial feature of the analysis presented in this paper.

The intrinsic quantum mechanical spreading of the initial wavefunction $\left\langle\mathbf{r}_{2} ; t=t_{0} \mid \psi\right\rangle$ during the acceleration by the laser field can be readily treated within its internal coordinate system $\mathbf{r}_{2}$ in the single non-interacting electron limit. This is because we expect the spreading of $|\psi\rangle$ to be essentially radial since $\left\langle r_{2} ; t=0 \mid \psi\right\rangle$ originated from a bound state $\left|\phi_{j}\right\rangle$ in a central potential. In other words, the symmetry dictated by the spherical harmonic $Y_{l m}(\theta, \phi)$ of the ionized state $\left|\phi_{j}\right\rangle$ is predominantly preserved in $\left\langle\mathbf{r}_{2} ; t \mid \psi\right\rangle$ since no torque is applied. Thus, to a first-order approximation, the time evolution of $|\psi\rangle$ may be written as [35]

$$
\left\langle\mathbf{r}_{2} ; t \mid \psi\right\rangle=\left\langle r_{2}(t), \theta, \phi \mid \psi\right\rangle ; r_{2}(t) \approx r_{20}\left(1+\frac{t^{2}}{\tau^{2}}\right)^{-\frac{1}{2}},
$$

where $r_{20}$ is the original radial coordinate and $\tau=m a^{2} / \hbar$ is the characteristic time for the radialwidth $a$ of the wavefunction's initial probability distribution to increase to $\sqrt{2}$ of its initial value. For example, the wavefunction for an electron photoionized from the $\mathrm{Xe} 4 p$ state, which has a 
bound radius of $\sim 0.7 \AA$, will double its width in $\sim 0.07 \mathrm{fs}$, i.e. in a fraction of a femtosecond! More significantly, when the photoionized Xe $4 p$ electron returns to collide with its cluster of origin after one 0.8 fs period of the $248 \mathrm{~nm}$ radiation, $|\psi\rangle$ has expanded to a radius of $\sim 14 \AA$. This is to be compared to the typical $\sim 10 \AA$ diameter 10-20 atom Xe cluster size used as the target material in the experiments generating the observed $\mathrm{Xe}(\mathrm{L})$ emission shown in Fig. 1. Thus, since the photoionized electron trajectory at irradiances of $10^{18}-10^{19} \mathrm{~W} / \mathrm{cm}^{2}$ is known to come back through the cluster near its center [25], the expanded wavefunction is optimally sized to interact with all the atoms in the Xe cluster. This optimal geometrical coupling condition for collisional ionization of inner-shell electrons from the Xe atoms in the cluster will be degraded at longer laser excitation wavelengths. For example, upon return to the target atoms in the cluster under $800 \mathrm{~nm}$ IR laser excitation (optical period of $2.7 \mathrm{fs}$ ), $|\psi\rangle$ would be $\sim 3$ times broader so that its central probability amplitude $\left\langle\mathbf{r}_{2} \approx 0 \mid \psi\right\rangle$ is reduced by a factor of 5-6. In turn, this should result in more than an order of magnitude reduction in the cross-section for the collisional ejection of any bound state $\left|\phi_{j}\right\rangle$ from the a Xe target atom/ion.

In addition to the intrinsic quantum mechanical spreading, the photoionized wavefunction $|\psi\rangle$ will also be dephased (i.e. loose its initial coherence) by collisional scattering. For sufficiently strong ATI, where all electrons in an entire orbital are simultaneously ionized [42], the dominant scattering mechanism will be internal electron-electron collisions within the photoionized state $\left\langle\mathbf{r}_{2} ; t \mid \psi\right\rangle$. Classically, this process results in a "Coulomb explosion" of the photoionized electron distribution [25] which converts of the shape of the initial coherent wavefunction $\left\langle\mathbf{r}_{2} ; t=0 \mid \psi\right\rangle$ into incoherent spherical waves originating, on average, from the center of the ionized electron distribution. In other words, this process is primarily responsible for the transition into the regime where a plane-wave analysis of the collisional ejection of inner-shell electrons becomes valid.

We may estimate the electron-electron scattering rate $\gamma_{\mathrm{ee}}$ for this dephasing mechanism by comparison with the large body of experimental work on carrier-carrier collisions in semiconductors. In particular, the observations of Portella et al. [20] and Becker et al. [21] indicate that the electron-electron scattering rate in bulk $\mathrm{GaAs}$ increases with the cube root of the carrier density, i.e. $\gamma_{\mathrm{ee}} \approx A_{\mathrm{GaAs}} n^{0.33}$ where $A_{\mathrm{GaAs}} \approx 3.7 \times 10^{5} \mathrm{~m} / \mathrm{s}$. Adjusting for the effective electron mass of GaAs ( $m^{*}=0.067 m_{0}$ [46], where $m_{0}$ is the free electron rest mass), we obtain a value of $\sim 1 \AA /$ fs for $A_{0}$ in a vacuum. Thus, for the maximal simultaneous photoionization by ATI of all six electrons of the $\mathrm{Xe} 4 p$ state in an initial volume with a radius of $-0.7 \AA$, we would expect an initial scattering rate of around $1-2 \times 10^{15} \mathrm{~s}^{-1}$, corresponding to a characteristic electron-electron 
scattering time of $0.5-1 \mathrm{fs}$. This initial scattering time is roughly a factor of 5 longer than the characteristic time associated with the intrinsic quantum mechanical spreading of the photoionized wavefunction. Consequently, the $4 p$ wavefunction photoionized from a solitary Xe ion spreads faster than it dephases. This means that it is possible for a significant fraction of the photoionized $4 p$ state to remain coherent during the ponderomotive acceleration over one radiation period before returning to interact with its parent Xe cluster.

The fraction $f$ of the photoionized state $\left\langle\mathbf{r}_{2} ; t \mid \psi\right\rangle$ that remains coherent as a function of time may be estimated by incorporating the reduction in the rate of internal electron-electron collisional dephasing caused by the spreading of the $|\psi\rangle$ into a rate equation of the form

$$
\frac{\partial f}{\partial t}=-A_{0} n_{0}^{0.33} f\left(1+\frac{t^{2}}{\tau^{2}}\right)^{-\frac{1}{2}}
$$

where $n_{0}$ is the initial electron density of the photoionized wavefunction. The solution to this equation, subject to the initial generation by ATI of a totally coherent state (i.e. $f=1$ at $t=0$ ), is

$$
\ln f=-A_{0} n_{0}^{0.33} \tau \ln \left[\frac{t}{\tau}+\sqrt{1+\frac{t^{2}}{\tau^{2}}}\right]
$$

For the photoionized $\mathrm{Xe} 4 p$ orbital with six electrons initially in a radius of $\sim 0.7 \AA$, this analysis indicates that the ionized state $\left\langle r_{2} ; t \mid \psi\right\rangle$ will be $30-40 \%$ coherent when it returns to interact with its cluster of origin after the $0.8 \mathrm{fs}$ radiation period of $248 \mathrm{~nm}$ laser excitation [25]. As a result, an accurate description of the collisional ejection mechanism generating the spectrally observed $2 p$ vacancies (Fig. 1) must incorporate a non-plane-wave component. Such considerations may even be necessary for $800 \mathrm{~nm}$ IR irradiation since equation (9) suggests that the $4 p$ orbital is still 20 $30 \%$ coherent after the longer $2.7 \mathrm{fs}$ radiation period, even though $\left\langle\mathbf{r}_{2} ; t \mid \psi\right\rangle$ is now substantially more spread out. However, this intra-orbital analysis does not consider dephasing due to interorbital electron-electron scattering which will begin to contribute to the decoherencing of $\left\langle\mathbf{r}_{2} ; t \mid \psi\right\rangle$ once the expanding photoionized states from different atoms in the cluster begin to overlap. The scattering rate due to this inter-orbital interaction is estimated to be $0.5-1 \mathrm{fs}^{-1}$, which suggests that the electron wavefunction may be almost completely dephased upon returning to interact with its cluster of origin when the driving radiation period exceeds $\sim 2 \mathrm{fs}$, i.e. for pumplaser wavelengths greater than $\sim 600 \mathrm{~nm}$. 


\section{III.C. Angular Momentum Selectivity in Collisional Ejection Mechanism}

The above analysis of the temporo-spatial dynamics of the photoionized wavefunction clearly indicates that inner-shell impact ionization due to incident Xe $4 p$ electrons under $248 \mathrm{~nm}$ laser excitation could be primarily affected by the residual coherence of the spreading $\left\langle\mathbf{r}_{2} ; t \mid \psi\right\rangle$ rather than plane-wave collisions resulting from a dephased $\left\langle\mathbf{r}_{2} ; t \mid \psi\right\rangle$. Accordingly, in the rest of this paper we will examine the physics behind such a coherent "orbital"-orbital collisional ionization process $[38,39]$.

With a knowledge of the form of the expanding and ponderomotively-driven photoionized wavefunction $\left\langle\mathbf{r}_{2} ; t \mid \psi\right\rangle$ given in equation (7), the essential physics behind the matrix element $M_{f i}$ associated with the collisional ejection of an electron in the bound state $\left\langle\mathbf{r}_{1} \mid \phi_{j}\right\rangle$ by the photoionized electron state may be readily determined for the case when $\mathbf{R}=0$. In this limit, equation (4) reduces to

$$
M_{f}(\mathbf{R}=0)=\iint d^{3} \mathbf{r}_{1} d^{3} \mathbf{r}_{2} \frac{\phi_{j}\left(\mathbf{r}_{1}\right) \psi\left(\mathbf{r}_{2}, t\right)}{r_{1} r_{2}\left|\mathbf{r}_{1}-\mathbf{r}_{2}\right|} \exp \left[-i \mathbf{k}_{1} \cdot\left(\mathbf{r}_{1}-\mathbf{r}_{2}\right)\right]
$$

which may be solved analytically using the expansion [47]

$$
\frac{e^{i \kappa\left|r_{1}-r_{2}\right|}}{4 \pi\left|\mathbf{r}_{1}-\mathbf{r}_{2}\right|}=i \kappa \sum_{L=0}^{\infty} j_{L}\left(\kappa r_{<}\right) h_{L}^{(1)}\left(\kappa r_{>}\right) \sum_{M=-L}^{L} Y_{L M}^{*}\left(\theta_{2}, \phi_{2}\right) Y_{L M}\left(\theta_{1}, \phi_{1}\right)
$$

where $j_{L}(\kappa r)$ are spherical Bessel functions of order $L, h_{L}^{(1)}(\kappa r)$ are Hankel functions of the first kind of order $L$, and $k=-k_{1} \cos \beta$ with $\beta$ equal to the angle between $k_{1}$ and the vector $r_{1}-r_{2}$. As with other collisional interactions [35,37], the matrix element $M_{\hat{f}}$ is a function of the details of the collision dynamics, that is $\mathbf{k}_{1}$ through $\mathbf{k}$.

We will begin by considering the collision physics associated with the angular contribution to $M_{f}(\mathbf{R}=0)$ in the integrand of equation (10). Here, it is important to note that, unlike the direction of $\mathbf{r}_{1}-\mathbf{r}_{2}$, the direction of the ejected electron's momentum $\hbar \mathbf{k}_{1}$ cannot be explicitly related to the integration variables variables $\theta_{1}, \phi_{1}, \theta_{2}$, and $\phi_{2}$. Instead, the allowed directions of $\mathbf{k}_{1}$ are determined solely by the momentum and energy conservation in the collision. As a result, 
the angle $\beta$ between $\mathbf{k}_{1}$ the vector $\mathbf{r}_{1}-\mathbf{r}_{2}$ must be considered as a random variable in the evaluation of $M_{f i}(\mathbf{R}=0)$ so that, for example, the energy conservation condition for the ejection of an innershell electron with an ionization potential $I_{\mathrm{p}}$ may be written as

$$
k_{0}^{2}-\frac{2 m I_{p}}{\hbar^{2}}=k_{2}^{2}+2 \kappa^{2}
$$

since, on average, $\cos ^{2} \beta=\frac{1}{2}$. It is then manifest that the form of the angular integrations involved in $M_{f i}$ imply a strong angular momentum selectivity to the collisional ejection of innershell electrons. Specifically, if one is interested in the collisional ejection of an electron from a bound state $\left|\phi_{j}\right\rangle$ with an orbital angular momentum quantum number $l$, then the insertion of equation (11) into equation (10) ensures that only the $L=l$ term of the Coulomb expansion contributes to $M_{f i}$ (the $\theta_{1}$ integration), and thence, that the collisional cross-section is non-zero only if the incident photoionized electronic state $\left\langle\mathbf{r}_{2} ; t \mid \psi\right\rangle$ has an angular momentum component of $l \hbar$ in the collision (the $\theta_{2}$ integration). Alternatively, if the ponderomotively-driven incident wavefunction $\left\langle\mathbf{r}_{2} ; t \mid \psi\right\rangle$ is predominantly a state with a particular orbital angular momentum quantum number, then strong $l$-state selectivity will result in the collisional ejection mechanism.

This type of $l$-state selectivity in the collisional ionization process is clearly in agreement with the observation of strong Xe $\mathrm{L}$-shell emission from $\mathrm{Xe}^{-30+}$ ions under intense $10^{18}-10^{19} \mathrm{~W} / \mathrm{cm}^{2} \mathrm{UV}$ laser excitation (Fig. 1). In particular, unlike the plane-wave collisional analysis, it explains the generation of $2 p$ vacancies in Xe ions which have all their $3 s$ and a substantial fraction of their $3 d$ electrons remaining. This is because, for laser irradiances $\geq 2.5 \times 10^{18} \mathrm{~W} / \mathrm{cm}^{2}$, sufficiently strong ATI can simultaneously photoionize the entire $\mathrm{Xe} 4 p$ shell to generate an initial 6-electron state of the form

$$
\left\langle\mathbf{r}_{2} ; t=0 \mid \psi\right\rangle=r_{2} R_{41}\left(r_{2}\right) \sum_{m=-1}^{1} Y_{1 m}\left(\theta_{2}, \phi_{2}\right)
$$

After approximately one period of the driving laser radiation, the time-evolving state $\left\langle\mathbf{r}_{2} ; t \mid \psi\right\rangle$ returns to interact with the Xe atoms in its cluster of origin [25]. Under $248 \mathrm{~nm}$ UV laser excitation with a $0.8 \mathrm{fs}$ radiation period, the $30-40 \%$ of the returning wavefunction $\left\langle r_{2} ; t \mid \psi\right\rangle$ which is still coherent with its initial photoionized $4 p$ state $\left\langle r_{2} ; t=0 \mid \psi\right\rangle$ will predominantly collisionally eject a bound electron from a state with the same spherical harmonic $Y_{I m}(\theta, \phi)$, i.e. a $p$-state electron with $l=1$. The matrix elements with all other bound states $\left|\phi_{j}\right\rangle$ with $l \neq 1$ will be 
severely supressed since the spreading of $\left\langle\mathbf{r}_{2} ; t \mid \psi\right\rangle$ has mostly preserved its $p$-wave character. For longer radiation periods, such as the $\approx 3 \mathrm{fs}$ period of near IR laser radiation, this $l$-state selectivity in the collisional ejection process is expected be sharply reduced by intra- and inter-orbital electron-electron scattering which destroy the symmetry of the initial photoionized state $\left\langle\mathbf{r}_{2} ; t=0 \mid \psi\right\rangle$, thus rapidly and progressively returning the inner-shell ejection process to the customary plane-wave regime. However, the coherent nature of the collisional interaction may not be completely destroyed, as evidenced by the observation of weak, yet identifiable, L-shell emission from Xe ions with charge states between $27+$ and $29+$ (Fig. 1); i.e. Xe ions with filled $3 s$ and mostly filled $3 d$ orbitals.

\section{III.D. Multiple Inner-Shell Vacancy Production}

It is interesting to note that, in addition to the $l$-state selectivity, $m$-state selectivity is also expected in the coherent inner-shell collisional ejection mechanism. This arises because the strong electric field from the exciting laser radiation imposes a preferred axis to the interaction through the Stark effect. As a result, the angular integrations over $\phi_{1}$ and $\phi_{2}$ ensure that a non-zero matrix element $M_{f}(\mathbf{R}=0)$ for the collisional ejection of an electron from a particular bound state $\left|\phi_{j}\right\rangle$ exists only when the incident photoionized state $\left\langle\mathbf{r}_{2} ; t \mid \psi\right\rangle$ has a component of the same azimuthal angular momentum as $\left|\phi_{j}\right\rangle$. Since the Coulomb interaction is spin invariant, this has an important consequence for the shell-selective collisional ejection process: namely, that it is possible for the two electrons in the same $l$ - and $m$-state of $\left\langle\mathbf{r}_{2} ; t \mid \psi\right\rangle$ to act collectively (or coherently) $[28,33,34]$ to collisionally eject two inner-shell electrons with the identical orbital angular momentum and magnetic quantum numbers. Such a collision between two correlated anti-symmetric spin states of the form $\left.\frac{1}{\sqrt{2}}\left(\frac{1}{2},-\frac{1}{2}\right\rangle-\left|-\frac{1}{2}, \frac{1}{2}\right\rangle\right)$ in the target and photoionized wavefunctions has the same value of the matrix element $M_{f i}$ as the single electron-electron collisional ejection mechanism in the absence of dephasing effects. Thus, the generation of double $m$-state vacancies by this selective coherent process is only 4 times less likely than the production of single vacancies - there being 4 possible single electron-electron collisions for each $l$ - and $m$-state selective interaction. This ratio of single to double vacancy production is to be contrasted to a ratio of $10^{3}-10^{4}$ for the collision of high $Z$ atoms ' $(Z>20)$ with high energy quanta [48]. This difference is expected since the coherent ejection mechanism is more reminiscent of the orbital-orbital interactions of ion-atom collisions $[38,39]$.

At first glance, one would expect the collision producing a double vacancy to be more affected by dephasing effects than the single-vacancy-generating interaction since, for the former, both 
electrons in the particular $m$-state of $\left\langle\mathbf{r}_{2} ; t \mid \psi\right\rangle$ must remain coherent. However, to a good first approximation, this is not the case within a single photoionized orbital, because the most likely electron-electron collision will occur between electrons in the closest spatial proximity; that is, between the spin pair in the same $m$-state wavefunction. Clearly, this dominant collisional dephasing process will have an equal effect on the rates of both single and double vacancy production, resulting in an approximately fixed ratio of single to double vacancy production of 4 . For atomic cluster or solid targets, the overlap between expanding photoionized wavefunctions from neighboring atoms or ions, and consequent inter-orbital dephasing collisions, will cause this ratio to increase. However, the experimental evidence of Fig. 1 indicates that the ratio of the peak emission strengths from Xe ions with single and double $2 p$ vacancies is only $\sim 5$, suggesting that the inter-orbital scattering rate may be less than $0.5 \mathrm{fs}^{-1}$ and thus not a strong effect for shortperiod UV laser excitation. The observation of strong double $2 p$ vacancy production in Xe clusters excited by $248 \mathrm{~nm}$ radiation, therefore, provides direct evidence for a coherent (or correlated) collisional ejection mechanism. Moreover, the lack of the same spectral signiture under $800 \mathrm{~nm}$ IR laser excitation, where the longer radiation period only serves to further "mix" and dephase the expanding ionized states $\left\langle\mathbf{r}_{2} ; t \mid \psi\right\rangle$, is clearly consistent with such as mechanism.

\section{III.E. Radial Selectivity in the Matrix Element}

To fully account for the experimentally observed anomalous Xe L-shell emission spectra (Fig. 1), the proposed coherent inner-shell ionization mechanism must also display significant $n$-state (or radial) selectivity in addition to the strong angular momentum selectivity discussed above. Specifically, it must explain the observed lack of strong $3 p$ ejection when the incident ponderomotively-driven state $\left\langle r_{2} ; t \mid \psi\right\rangle$ originated from a photoionized $4 p$ orbital at $\mathrm{UV}$ irradiances of $10^{18}-10^{19} \mathrm{~W} / \mathrm{cm}^{2}$. This follows because the experimental designation and calculational verifications of the charge states in the observed $\mathrm{Xe}(\mathrm{L})$ emission from $2 p$ vacancies support the conclusion that the $3 p$ shell is intact. In other words, the collisional cross-section for the ejection of a $2 p$ electron by the proposed mechanism should be greater than that for the ejection of a $3 p$ electron, a result that stands in contradiction to the conventional plane-wave collisional analysis (Fig. 2). Since this type of selectivity cannot be $l$ or $m$ dependent, it must be due to radial dependence of the matrix element $M_{\hat{f}}(\mathbf{R}=0)$.

Evaluation of the radial contribution to the matrix element $M_{f i}$ for $\mathbf{R}=0$ requires knowledge of the radial forms of both the initially ionized wavefunction $\left\langle\mathrm{r}_{2} ; t=0 \mid \psi\right\rangle$ and the bound states $\left|\phi_{j}\right\rangle$ of the target ion. In order to simplify this calculation, we will approximate these states by suitably 
scaled (to account for screening) hydrogenic wavefunctions [35]. Figure 4 compares the computed normalized hydrogenic wavefunctions $r R(r)$ (dashed curves) with those obtained from an exact calculation (solid curves) [49] for the $2 p, 3 p$, and $4 p$ radial orbitals of atomic Xe. The relatively good agreement in all three cases allows the matrix element $M_{f i}(\mathbf{R}=0)$ to be evaluated analytically to a high precision. The slight disagreement on the form of the outer lobe of the $4 p$ orbital accounts for less than a $2 \%$ error in the calculation, since $(i)$ the $1 / \mathrm{r}$ dependence of $\mathrm{V}_{\mathrm{c}}$ supresses its contribution relative to the first two lobes and (ii) the overlap of the outer lobe of the $4 p$ state with the $3 p$ and $2 p$ orbitals is minimal. Moreover, the rapid radial expansion of the $4 p$ orbital after ATI ensures that effectively only the inner two of its well-represented radial lobes contribute to the matrix element after a time $t>2 \tau \approx 0.14 \mathrm{fs}$. In fact, for small laser-excited Xe clusters, where the photoionized electrons return to interact with their cluster of origin after one radiation period (i.e. after a time $t / \tau>10$ ) [25], essentially only the first radial lobe of the expanding $4 p$ orbital overlaps with both the bound $2 p$ and $3 p$ states for $\mathbf{R}=0$. In addition, it should be noted that the use of the radial forms of the $2 p, 3 p$, and $4 p$ states for a Xe atom rather than an $\mathrm{Xe}^{+q}$ ion of the appropriate charge should not affect the evaluation of the matrix element significantly. This is because the basic shape of both the tightly-bound inner-shell $2 p$ and $3 p$ states and the innermost first two lobes of the $4 p$ state, which dominate the collisional ejection mechanism, should not be significantly perturbed for typical charge states of $q \approx+30$ generated at the experimental excitation irradiances of $10^{18}-10^{19} \mathrm{~W} / \mathrm{cm}^{2}$.

The result of evaluating the radial integration of the matrix element for the collisional interaction in equation (8) using the hydrogenic $2 p, 3 p$, and incident expanding $4 p$ wavefunctions is shown in Figure 5. The value of $\left|M_{f i}(\mathbf{R}=0)\right|^{2}$, which is directly proportional to the collisional cross-section for the selective ejection mechanism, is plotted as a function of the normalized time $t / \tau$ given by equation(7) and $\kappa=k_{1} \cos \beta$ for the collisional ejection of the bound $2 p$ and $3 p$ states in Figs. 5(a) and (b) respectively. Three features are readily apparent. First, in contrast to the plane-wave collisional analysis (Fig. 2) [32], the cross-section for $3 p$ ejection is less than that for the impact ionization of a $2 p$ electron at equivalent values of $t / \tau$ and $\mathrm{K}$. Second, as expected, the crosssection for the impact ionization of inner-shell electrons decreases in time due to the reduction in the collisional overlap integral caused by the temporal spreading of the photoionized state $\left\langle\mathbf{r}_{2} ; t \mid \psi\right\rangle$. Third, the cross-section is also a strong function of $\mathrm{k}$ with slower, less energetic collisions being favored for $t / \tau \geq 10$. Physically, the latter trend may be understood in terms of the collision time - a less energetic collision allowing for a longer time period for the wavefunctions representing the electron states to interact. Mathematically, it is a direct consequence of the form of the Bessel function product $j_{1}(\mathrm{kr}) h_{1}^{(1)}(\mathrm{kr})$ in equation (11) which. 
for sufficiently large values of $k$, has a more rapid oscillation with $r$ than the $2 p$ and $3 p$ bound state wavefunctions (Fig. 4), thus reducing the value of $\left|M_{f i}(\mathbf{R}=0)\right|^{2}$.

To quantify the first two observations, we will examine in more detail the interaction conditions leading to the L-shell spectra of Fig. 1 emitted by Xe clusters under $248 \mathrm{~nm}$ UV and $800 \mathrm{~nm}$ IR laser excitation. Form the relativistic analysis of Ref. 25 , a photoionized electron state $\left\langle\mathbf{r}_{2} ; t \mid \psi\right\rangle$ will return to its cluster of origin with a collision energy of $\sim 15 \mathrm{keV}$ after approximately one radiation period for both pump-laser wavelengths at the experimental irradiances of $10^{18_{-}}$ $10^{19} \mathrm{~W} / \mathrm{cm}^{2}$. With this information, the most probable average value of $\kappa$ for an inner-shell ejection process may be calculated using equation (12) and assuming the equipartition of the energy available after the collision between the scattered and ejected electrons. For the ejection of a $2 p$ electron with an ionization potential $I_{\mathrm{p}} \sim 8 \mathrm{keV}$, this calculation gives $\kappa \approx 21 \AA^{-1}$, whereas for $3 p$ vacancy production we have $\mathrm{k} \approx 28 \AA^{-1}$ since $I_{\mathrm{p}} \sim 2.8 \mathrm{keV}$ in this case. For these values of $\kappa$, Figure 6(a) displays the temporal dependence of $\left|M_{f i}(\mathbf{R}=0)\right|^{2}$ for the ejection of both $2 p$ (solid line) and $3 p$ (dotted line) electrons by an interaction with the incident expanded and coherent $4 p$ state.

Most notably, Fig. 6(a) clearly indicates that the cross-section for the collisional ejection of a $2 p$ electron by the ponderomotively-driven and expanding $4 p$ orbital is always at least two orders of magnitude greater than for the ejection of a $3 p$ electron. This additional radial or $n$-state selectivity is a direct consequence of the shape of the $3 p$ radial wavefunction relative to that of the $2 p$ state; namely, the bipolar nature of the $3 p$ orbital supresses the matrix element associated with the collisional interaction. In other words, the ejection of electrons from states with the fundamental single-peaked radial wavefunction is strongly preferred in this selective coherent interaction. As shown by the plot in Fig. 6(b), this is in fact true for all values of $\kappa$ and, hence, incident collision energies for the $4 p$ state. Moreover, the ratio of $2 p$ to $3 p$ collisional crosssections for this coherent interaction is also relatively insensitive to the excitation wavelength for $\kappa>5 \AA^{-1}$. This result is clearly consistent with the observed $X e(L)$ emission under intense laser excitation, because virtually no $3 p$ vacancy generation would be expected even for multiple $2 p$ vacancy production; an outcome in agreement with the charge labeling in Fig. 1 for both the IR and UV excitation wavelengths. Furthermore, the calculated ratio between the $2 p$ and $3 p$ collision cross-section represents at least a three orders of magnitude reversal over the crosssection ratio evaluated from a plane-wave analysis (Fig. 2) [32].

Figure 6(a) also reveals a strong pump-laser wavelength dependence to the selective collisional ionization process; namely, that the coherent orbital-orbital collision is much more probable for 
shorter pump-laser wavelengths (i.e. short radiation periods). Specifically, since $\tau \approx 0.04 \mathrm{fs}$ for the $4 p$ Xe state with an initial bound-state radius $a$ of $\sim 0.7 \AA$, the value of $\left|M_{f i}(\mathbf{R}=0)\right|^{2}$ for the ejection of a $2 p$ electron is $\sim 350$ times greater under $248 \mathrm{~nm}$ UV laser excitation (collision at $t / \tau=$ 19) than for the $800 \mathrm{~nm}$ pump-laser wavelength (collision at $t / \tau=63$ ). The intrinsic quantum mechanical spreading of the photoionized $4 p$ state causes a similar reduction in the cross-section for $3 p$ impact ionization as the excitation wavelength increases. The inclusion of collisional dephasing within the incident photoionized state $\left\langle\mathbf{r}_{2} ; t \mid \psi\right\rangle$, which destroys the selectivity of the inner-shell ejection process, further magnifies this adverse dependence on the radiation period. For an estimated average electron-electron scattering rate of the order of $1 \mathrm{fs}^{-1}$ (Section III.B), we expect a ratio in the selective collisional cross-sections of $\sim 10^{3}$ favoring the use of $248 \mathrm{~nm} \mathrm{UV}$ over $800 \mathrm{~nm}$ IR laser excitation. This cross-section ratio is in good agreement with the experimental observation of a strong pump-laser wavelength dependence in the Xe L-shell emission $[24,25]$ which indicates that the shorter-period UV radiation is $\sim 3000$ times more effective at generating $2 p$ vacancies in 5-20 atom Xe cluster targets than equivalent terawatt $\mathbb{R}$ $(800 \mathrm{~nm})$ radiation. An exact calculation of all collisional dephasing rates in expanding photoionized states $\left\langle\mathbf{r}_{2} ; t \mid \psi\right\rangle$ and a full evaluation of the matrix element $M_{f i}$ integrated over all values of $\mathbf{R}$ in a more rigorous analysis, both of which are beyond the scope of this paper, may yield a closer agreement with the experimental results. Nevertheless, the approximate treatment unambiguously shows that the wavelength dependence is strong and that the use of sufficiently long pump-laser wavelengths will eliminate the selectivity of the interaction.

It is intersting to note that since the $3 p$ cross-section is at least one order of magnitude less than the $2 p$ cross-section for timescales $t / \tau<5$, it may be possible to circumvent the strong pump-laser wavelength dependence of $\mathrm{L}$-shell emission by ensuring that the time between the photoionization by ATI and the collisional ejection is much less than the period of the driving radiation. This immediately implies that the ionized state $\left\langle\mathbf{r}_{2} ; t \mid \psi\right\rangle$ must interact within the first quarter of the laser radiation period with an atom/ion that is remote from its atom of origin as could be the case in a solid target material or in large $(>100)$ atom clusters. However, for the selective mechanism to be effective, the laser-driven state $\left\langle\boldsymbol{r}_{2} ; t \mid \psi\right\rangle$ cannot be significantly dephased by collisions with other atoms/ions in the target material prior to reaching the incident kinetic energy necessary for the collisional ejection of a particular inner-shell electron. Such a condition is expected to be difficult to achieve unless the ponderomotive potential is sufficiently strong to allow acceleration to the required kinetic energy over inter-atomic distances. Nonetheless, precisely this type of interaction has already been observed in the generation of $2 \sigma_{\mathrm{g}}$ vacancies in $\mathrm{N}_{2}$ [50] and in the more recent detection of strong $\mathrm{Ba}(\mathrm{L})$ emission from solid $\mathrm{BaF}_{2}$ targets [51] under excitation with intense sub-picosecond $248 \mathrm{~nm}$ radiation. 


\section{Inner-Shell Population Inversion}

The selective collisional ionization mechanism described above is clearly well suited to the efficient generation of inner-shell vacancies and, hence, the population inversion required for the development of coherent kilovolt $\mathrm{x}$-ray radiation sources. In fact, given the quantum state degeneracies of the $2 p$ and $3 d$ levels, the experimental observation (Fig. 1) of anomalously strong $\mathrm{Xe}(\mathrm{L})$ emission due to $2 p$ vacancies from both $\mathrm{Xe}^{27+}\left(2 p^{5} 3 d^{10}\right)$ and $\mathrm{Xe}^{28+}\left(2 p^{5} 3 d^{9}\right)$ is already direct evidence for the efficient generation of inner-shell-inverted ionic species in laser-driven plasmas. Whether or not population inversion can be produced is dependent upon the relative rates of generation of the upper and lower states of the considered transition; for example, the electron configurations $2 p^{5} 3 d^{10}$ and $2 p^{6} 3 d^{9}$ respectively for L-shell emission from $\mathrm{Xe}^{27+}$. Nonetheless, the above analysis of the experimental results (Fig. 1) indicates that a UV laserpumped Xe L-shell system has great potential as a coherent $3 \AA(\approx 4 \mathrm{keV}) \mathrm{x}$-ray radiation source. Furthermore, the strong relativistic self-focusing effects observed in Xe cluster targets under $248 \mathrm{~nm}$ pump-laser irradiation [52] may produce an optimal guided-channel geometry [29,40] extending over several Rayleigh ranges for both the optical pumping and the $x$-ray emission.

The extension of this collisional process to the generation of more energetic $\mathrm{x}$-ray radiation would require the use of either higher $Z$ elements for the equivalent $\mathrm{L}$-shell emission or $\mathrm{K}$-shell emission through the $1 s^{2} 2 p^{5} \leftarrow 1 s 2 p^{6}$ transition in elements with $Z>30$. While the former is complicated by the engineering of suitable metal cluster or other composite and compound (e.g. UF 6 ) targets, the latter possibility appears to offer more practical flexibility. A similar selective collisional ionization process for K-shell emission would require the photoionization by ATI and subsequent ponderomotive acceleration of the $3 s$ state to collisionally eject an electron from the $1 s$ state within one radiative period. Unfortunately, for $\mathrm{Xe}(\mathrm{K})$ emission, a very high laser irradiance of $-3 \times 10^{20} \mathrm{~W} / \mathrm{cm}^{2}$ to ionize the $3 s$ state would be required. A attractive alternative target atomic species, which could produce very useful $\sim 1 \AA \mathrm{K}$-shell radiation [13], is $\mathrm{Kr}$ for which ATI of the $3 s$ state requires a laser irradiance of $\sim 2 \times 10^{19} \mathrm{~W} / \mathrm{cm}^{2}$ - a value associated with the channeling of $248 \mathrm{~nm}$ radiation in underdense plasmas [29].

Figures $7(a)$ and (b) show, respectively, the results of evaluating the modulus-square of the explicit $l$-state selective matrix element, i.e. $\left|M_{f_{2}}(R=0)\right|^{2}$, for the collisional ejection of bound $1 s$ and $2 s$ electrons in $\mathrm{Kr}$ by ponderomotively-driven $3 s$ electrons as a function of $\mathrm{K}$ and the normalized time $t / \tau$ for the expanding photoionized orbital. Again, the collision cross-section 
decreases in time, due to the spreading ionized $3 s$ wavefunction, and with increasing collision energy, i.e. K. However, in this case, it is not immediately apparent whether it is more probable to collisionally eject a $1 s$ or a $2 s$ electron in the coherent interaction. The answer to this question lies in the details of the collision dynamics.

At a laser irradaince of $\sim 2 \times 10^{19} \mathrm{~W} / \mathrm{cm}^{2}$, the relativistic kinetic analysis of Ref. 25 indicates that an electron photoionized by ATI will return to a small ( 10 atom) $\mathrm{Kr}$ cluster with an energy of $\sim 20 \mathrm{keV}$ after approximately one radiation period. Since the ionization potentials of the $1 s$ and $2 \mathrm{~s}$ states are $\sim 17 \mathrm{keV}$ and $\sim 3.2 \mathrm{keV}$, the most probable average values for $\kappa$ are 14 and 33 for the ejected $1 s$ and $2 s$ electrons respectively. For these values of $\mathrm{k}$, the temporal dependence of $\left|M_{f i}(\mathbf{R}=0)\right|^{2}$ for the ejection of both $1 s$ (solid line) and $2 s$ (dotted line) electrons by an interaction with the ponderomotively-driven coherent $3 s$ state is shown in Figure 8 . It is immediately apparent that again the impact ionization of the inner single-lobed bound $1 s$ state is favored over the ejection of an electron from the $2 s$ state, but only by roughly one order of magnitude. The fact that the magnitudes of the two matrix elements are much closer for this $s$ state interaction than equivalent elements in the pervious $p$-state collisions is due to the form of the Laguerre polynomials for $l=0$; namely, that the relative width of the first radial lobe of the $s$ states is less than that of the $p$-states. Consequently, the overlap of the expanding photoionized $3 s$ wavefunction with the relatively large second lobe of the $2 s$ state dominates the matrix element despite the $1 / r$ variation of the Coulomb potential. Nevertheless, the analysis suggests that it may be possible to generate efficiently through laser excitation $\mathrm{Kr}$ ions exhibiting inversion between the $2 p$ and $1 s$ states without significant influence from a parasitic $2 s \leftarrow 2 p$ transition.

The efficiency with which such an inner-shell-inverted ionic species are produced is, however, again a strong function of the pump-laser wavelength due to the spreading of the ponderomotively-driven $3 s$ state over one radiation period. In this case, the smaller initial $\sim 0.5 \AA$ radius of the $\mathrm{Kr} 3 s$ orbital [49] ensures that the photoionized wavefunction spreads at almost twice the rate of a Xe $4 p$ orbital ionized by ATI. As a result, for $\mathrm{Kr}$ K-shell emission, the ratio of the $1 s$ collisional cross-sections at pump-laser wavelengths of $248 \mathrm{~nm}(t / \tau \approx 40)$ and $800 \mathrm{~nm}(t / \tau \approx$ 130 ) only favors the UV laser radiation by a factor of $\sim 30$ - an order of magnitude less than for the $2 p$ cross-section in the equivalent Xe L-shell process (Fig. 6(a)). However, the initial internal dephasing rate due to electron-electron scattering in the photoionized 2-electron $\mathrm{Kr} 3 s$ orbital is very close to that of the 6-electron Xe $4 p$ orbital ionized by ATI, since the initial electron densities are nearly identical. Consequently, after inclusion of collisional dephasing, the ratio between the 
two cross-sections is again expected to favor the shorter $248 \mathrm{~nm}$ pump-laser wavelength by a factor of at least 100 .

A comparison of the magnitudes of $\left|M_{f i}(\mathbf{R}=0)\right|^{2}$ evaluated for the generation of $\mathrm{Xe}(\mathrm{L})$ and $\mathrm{Kr}(\mathrm{K})$ emission by inner-shell vacancy production (e.g. Figs. 6(a) and 8) also suggests that the crosssection for $1 s$ ejection may be substantially larger than that for the impact ionization of a $2 p$ electron for this coherent interaction. This is predominantly due to the $r$ radial dependence of the bound atomic wavefunctions which suppresses the dominant contribution to the matrix element of the Coulomb interaction near the origin of the coordinate system (Fig. 3) when $l \neq 0$. However, this trend may be reversed in a full evaluation of the matrix element $M_{f}$, because the larger $2 p$ bound-state target wavefunction will contribute more to the interaction for $\mathbf{R} \neq 0$ than the smaller $1 s$ atomic orbital. Moreover, the $2 p$ ejection cross-section will be enhanced by an order of magnitude over the $1 s$ ejection cross-section since there are three more $m$-states (and electrons) in a full $p$-orbital than in a s-state. In addition, it should be noted that the relativistic kinematic analysis of Ref. 25 indicates that the Lorentz force in the ponderomotive potential will force the driven photoionized wavefunction $\left\langle\mathbf{r}_{2} ; t \mid \psi\right\rangle$ to avoid a collision with the target atom(s) in gas or atomic cluster materials at sufficiently high laser irradiances. Thus, since this effect is much less severe for shorter radiation periods (due to the reduced impulse), the future development of a coherent $\sim 1 \AA \mathrm{x}$-ray radiation source based on a gaseous target material strongly favors the use of high-power short wavelength (UV) rather than long wavelength (IR) pump lasers. In fact, the combined deleterious effects of the Lorentz force and the dephasing of $\left\langle\mathbf{r}_{2} ; t \mid \psi\right\rangle$ by electronelectron scattering may be the major limiting factors for the efficient generation of the inner-shell population inversion required for an atom-based coherent hard $\mathrm{x}$-ray radiation source.

\section{Conclusions}

The selective laser-driven collisional ionization mechanism outlined in this paper provides an explanation for the observation of spectrally anomalous and unexpectedly strong L-shell emission from atomic Xe clusters under intense irradiation $\left(10^{18}-10^{19} \mathrm{~W} / \mathrm{cm}^{2}\right)$ by sub-picosecond UV TW laser pulses at $248 \mathrm{~nm}$ (Fig. 1). In particular, it elucidates the physics behind the preferential generation of $\mathrm{Xe} 2 p$ vacancies in collisions with ponderomotively-driven $4 p$ electrons that are photoionized by the strong ATI expected in the observed stable channeled propagation of the UV laser radiation under relativistic self-focusing conditions $[29,40,52]$. Such a specific coupling of the incident laser energy into target atoms, at a rate that may exceed $1 \mathrm{~W} /$ atom $[22,23,53]$, could lead to the inner-shell population inversion required for the development of future coherent hard 
$\mathrm{x}$-ray sources. Indeed, the experimental observation of $\sim 5 \mathrm{keV} \mathrm{L}$-shell emission from both $\mathrm{Xe}^{27+}\left(2 p^{5} 3 d^{10}\right)$ and $\mathrm{Xe}^{28+}\left(2 p^{5} 3 d^{9}\right)$ already provides direct evidence that laser-driven inner-shell inverted ions can be efficiently generated. We note that the extension of this mechanism to uranium would generate L-shell emission in the $15-17 \mathrm{keV}$ range. Analysis of a similar selective collisional ejection process to produce $1 s$ vacancies in $\mathrm{Kr}$ suggests that the development of an efficient $\mathrm{K}$-shell radiation source may also be possible.

The analysis of this novel laser-driven collisional ionization mechanism also indicates an extraordinarily strong laser wavelength dependence for gas-phase-like targets (e.g. small atomic clusters and dilute gases) where the photoionized electrons may return to interact with the atom/cluster of origin after approximately one radiative period [25]. Both the intrinsic quantum mechanical spreading of the photoionized wavefunction and its dephasing by electron-electron scattering ensure that the efficiency of the selective collisional ejection process is reduced by a factor of $\sim 10^{3}$ as the pump-laser wavelength is increased by only a factor of $\sim 3$ from the UV $(248 \mathrm{~nm})$ to the $\mathbb{R}(800 \mathrm{~nm})$. In fact, for sufficiently long laser radiative periods, the dephasing causes the progression to the plane-wave scattering regime for which the relative cross-sections indicate that the generation of inner-shell population inversion for kilovolt $\mathrm{x}$-ray emission in high $Z$ atoms is ruled out. The analysis also shows that this impossibility is robust and cannot be overcome by the use of higher laser powers, such as petawatt Ti:sapphire- or Nd-based IR laser systems whose long radiation wavelength would essentially avoid the special features arising from the coherent phase dependence of the interaction. Form this point of view, the recent demonstration of an efficient third harmonic generation scheme for high power IR lasers appears very attractive [54]. The strong pump-laser wavelength dependence could be circumvented, while retaining the $l$-state and significant radial ( $n$-state) selectivity of the collisional ionization mechanism, by using condensed-phase targets (e.g. solid targets and large atomic clusters) provided that the ionization collisions occurred within a quarter of radiation period, i.e. the photoionized electron interacted with an atom other than its atom of origin. However, the selectivity of ionization process is likely to be rapidly impaired by scattering with ions and other electrons in the laser-generated plasma as the photoionized wavefunction is accelerated to the incident collisional energy necessary for inner-shell impact ionization.

A more elaborate and sophisticated calculation of the matrix element $M_{f i}$ for the kinematics of the selective collisional ionization mechanism should provide further insight into the conditions required for the optimally efficient generation of inner-shell vacancies. In particular, explicit evaluation of $M_{f i}$ for $\mathbf{R} \neq 0$ should allow the limits of the strength of the 1 -state selectivity of the mechanism to be determined. A comparison of the cross-section of this selective mechanism with 
respect to the plane-wave case is also required to quantify experimentally observed enhancement of the former. In addition, a more detailed evaluation of the effect of quantum mechanical spreading on the collisional dephasing dynamics internal to the photoionized wavefunction is necessary. Nevertheless, it is clear that the selective collisional ionization mechanism provides a new mode of energy coupling to atoms which could result in the efficient and controlled generation of inner-shell population inversion required of potential coherent kilovolt $\mathrm{x}$-ray sources. Furthermore, the evident consistency between the theoretical analysis and the experimental data strongly suggests that the pump-laser wavelength is a substantially more important parameter than the laser power or intensity.

\section{Acknowledgments}

The authors would like to thank Drs. T. Imbo and W. Pötz for useful discussions. The authors respectfully acknowledge the expert technical assistance of J. Wright and P. Noel during the experimental investigations and $\mathrm{M}$. Mehendale in the preparation of the manuscript. Support for this research was provided under grants and contracts with the National Science Foundation (BIR 9513266), SDI/NRL (N00014-93-K-2004), Army Research Office (DAAG55-97-1-0310), the Department of Energy at the Sandia National Laboratories and the United States Department of Energy (DE-AC04-94AL85000 and BB9131), the Japanese Ministry of Education, Science, Sport, and culture (\#08405009 and \#08750046) and the Murata Science Foundation. Sandia is a multi-program laboratory operated by Sandia Corporation, a Lockheed Martin company, for the the United States Department of Energy under contract DE-AC04-94AL85000. 


\section{FIGURE CAPTIONS}

\section{Figure 1}

The kilovolt Xe L-shell $(2 p \leftarrow-3 d)$ x-ray spectrum emitted by $5-20$ atom Xe clusters pumped by TW ultraviolet $(248 \mathrm{~nm})$ and infrared $(800 \mathrm{~nm})$ sub-picosecond laser pulses. The charge-state designations are the result of an analysis based on the work of Cowan [30], which also indicates that the feature at $2.6-2.65 \AA$ is attributable to the generation of multiple $2 p$ vacancies.

\section{Figure 2}

The plane-wave collision cross-sections $\sigma$ for the ejection of $2 p, 3 s, 3 p$, and $3 d$ electrons with ionization potentials of $8 \mathrm{keV}, 3.3 \mathrm{keV}, 2.8 \mathrm{keV}$, and $1.8 \mathrm{keV}$ respectively as a function of the energy of the incident colliding electron (after McGuire [32]). For convenience, the crosssections are normalized to the maximum cross-section for the ejection of a $2 p$ electron at a incident collision energy of $\sim 30 \mathrm{keV}$. The shaded region indicates the $10-20 \mathrm{keV}$ energy of photoionized electrons returning to interact with their cluster of origin for laser irradiances of $10^{18}-10^{19} \mathrm{~W} / \mathrm{cm}^{2}$.

\section{Figure 3}

The interaction geometry for the ejection of an electron from a bound state $\left\langle\mathbf{r}_{1} \mid \phi_{j}\right\rangle$ via a Coulombic collision with a ponderomotively-driven photoionized electronic state $\left\langle\mathbf{r}_{2} ; t \mid \psi\right\rangle$ displaced by $\mathbf{R}$ from the target atom (or ion).

\section{Figure 4}

Comparison of the exact (solid curves) and hydrogenic (dashed curves) normalized radial wavefunctions $r R(r)$ for the $2 p, 3 p$, and $4 p$ orbital of Xe. The hydrogenic wavefunctions are obtained using effective nuclear charges of 50,42 , and 40 respectively.

\section{Figure 5}

The calculated value of $\left|M_{\hat{l}}(\mathbf{R}=0)\right|^{2}$ as a function of the normalized expansion time $t / \tau$ and $\kappa=k_{1} \cos \dot{\beta}$ for the coherent collisional ejection of (a) $2 p$ and (b) $3 p$ electrons in $\mathrm{Xe}$ by an expanding photoionized $4 p$ state. 
Figure 6

a) The value of $\left|M_{f i}(\mathbf{R}=0)\right|^{2}$ as a function of the normalized expansion time $t / \tau$ for the collisional ejection of a $2 p$ electron with $\mathrm{\kappa}=21 \AA^{-1}$ (solid line) and a $3 p$ electron with $\mathrm{K}=$ $28 \AA^{-1}$ (dashed line) in Xe by a photoionized $4 p$ state. The vertical lines indicate the periods for $248 \mathrm{~nm} U$ UV and $800 \mathrm{~nm}$ IR radiation.

b) The ratio of $\left|M_{f i}(\mathbf{R}=0)\right|^{2}$ for $2 p$ versus $3 p$ inner-shell electron ejection as a function of $\kappa=k_{1} \cos \beta$ for a collision after one $0.8 \mathrm{fs} 248 \mathrm{~nm}$ radiation period (solid line) and one $2.7 \mathrm{fs} 800 \mathrm{~nm}$ radiation period (dashed line).

\section{Figure 7}

The calculated value of $\left|M_{f i}(\mathbf{R}=0)\right|^{2}$ as a function of the normalized expansion time $t / \tau$ and $\kappa=k_{1} \cos \beta$ for the coherent collisional ejection of (a) $1 s$ and (b) $2 s$ electrons in $\mathrm{Kr}$ by an expanding photoionized $3 s$ state.

\section{Figure 8}

The value of $\left|M_{f i}(\mathbf{R}=0)\right|^{2}$ as a function of the normalized expansion time $t / \tau$ for the collisional ejection of a $1 s$ electron with $\kappa=14 \AA^{-1}$ (solid line) and a $2 s$ electron with $\kappa=33 \AA^{-1}$ (dashed line) in $\mathrm{Kr}$ by a photoionized $3 s$ state. The vertical lines indicate the periods for $248 \mathrm{~nm}$ UV and $800 \mathrm{~nm}$ IR radiation. 


\section{RERFERENCES}

[1] McPherson A., Gibson G., Jara H., Johann U., Luk T.S., McKintyre I.A., Boyer K., and Rhodes C.K. 1987 Studies of multiphoton production of vacuum-ultraviolet radiation in rare gases J. Opt. Soc. Am. B 4595

[2] Zhou J, Peatross J., Murnane M., Kapteyn H., and Christov I. 1996 Enhanced highharmonic generation using 25 fs laser pulses Phys. Rev. Lett. 76, 752; Chang Z., Rundquist A., Wang H., Murnane M., and H. Kapteyn 1997 Generation of coherent soft x-rays at 2.7nm using high harmonics Phys. Rev. Lett. 792967

[3] Spielmann Ch., Burnett N.H., Sartania S., Koppitsch R., Schnürer M., Kan C., Lenzner M., Wobrauschek P., and Krausz F. 1997 Generation of coherent X-rays in the water window using 5-femtosecond laser pulses Science 278 661; Schnürer M., Spielmann Ch., Wobrauschek P., Streli C., Burnett N.H., Kan C., Ferencz K., Koppitsch R., Cheng Z., Brabec T., and Krausz F. 1999 Coherent 0.5-keV x-ray emission from helium driven by a sub-10-fs laser Phys. Rev. Lett. 803236

[4] Macchietto C.D., Benware B.R., and Rocca J.J. 1999 Generation of millijoule-level soft-xray laser pulses at a $4-\mathrm{Hz}$ repetition rate in a highly saturated tabletop capillary discharge amplifier Opt. Lett. 241115

[5] Matthews D. L., Hagelstein P. L., Rosen M. D., Eckart M. J., Ceglio N. M., Hazi A. U., Medscki H., MacGowan B. J., Trebes J. E., Whitten B. L., Cambell E. M., Hatcher C. W., Hawryluk A. M., Kauffman R. L., Pleasance D. L., Rambach G., Scofield J. H., Stone G., and Weaver T. A. 1985 Demonstration of a soft $\mathrm{x}$-ray amplifier Phys. Rev. Lett. 54, 110

[6] Zhang J., Key M. H., Norreys P. A., Tallents G. J., Behjat A., Danson C., Demir A., Dwivedi L., Holden M., Lewis C. L. S., MacPhee A. G., Neely D., Pert G. J., Ramsden S. A., Rose S. J., Shao Y. F., Thomas O., Walsh F., and You Y. L. 1995 Demonstration of high gain in a recombination XUV laser at $18.2 \mathrm{~nm}$ driven by a $20 \mathrm{~J}, 2$ ps glass laser Phys. Rev. Lett. 74, 1335

[7] Da Silva L. B., London R. A., MacGowan B. J., Mrowka S., Matthews D. L., and Craxton R. S. 1994 Generation of a 45-ps-duration 15.5-nm x-ray laser Opt. Lett. 19, 1532

[8] Lemoff B. E., Lin G. Y., Gordon III C. L., Barty C. P. J., and Harris S. E. 1995 Demonstration of a 10- $\mathrm{Hz}$ femtosecond-pulse-driven XUV laser at $41.8 \mathrm{~nm}$ in XE IX Phys. Rev. Lett. 74, 1574

[9] Kim D., Toth C., and Barty C.P.J. 1999 Population inversion between atomic inner-shell vacancy states by electron-impact ionization and Coster-Kronig decay Phys. Rev. A 59 R4129

[10] Duguay A. and Rentzepis P.M. 1967 Vacuum u.v. and x-ray lasers Appl. Phys. Lett. 10350 
[11] Moribayashi K., Sasaki A., and Tajima T. 1999 X-ray emission by ultrafast inner-shell ionization from vapors of Na, Mg, and $\mathrm{Al}$ Phys. Rev. A 592732

[12] Schoenlein R. W., Leemans W. P., Chin A. H., Volfbeyn P., Glover T. E., Balling P., Zolotorev M., Kim K.-J., Chattopadhyay S., and Shank C. V. 1996 Femtosecond x-ray pulses at $0.4 \AA$ generated by $90^{\circ}$ Thomson scattering: A tool for probing the structural dynamics of materials Science 274, 236; Leemans W. P., Schoenlein R. W., Volfbeyn P., Chin A. H., Glover T. E., Balling P., Zolotorev M., Kim K. J., Chattopadhyay S., and Shank C. V. $1996 \mathrm{X}$-ray based subpicosecond electron bunch charaterization using 90-degrees Thomson scattering Phys. Rev. Lett. 77, 4182

[13] Solem J. C. and Baldwin G. C. 1982 Microholography of living organisms Science 218, 229; Solem J. C. and Chapline G. F. 1984 X-ray biomicroholography Opt. Eng. 23, 3; Haddad W. S., Cullen D., Solem J. C., Boyer K., and Rhodes C. K. 1988 X-ray Fouriertransform holographic microscope Proceedings of the OSA Topical Meeting - Short Wavelength Coherent Radiation: Generation and Applications, p.284, North Falmouth, MA (Optical Society of America); Boyer K., Solem J. C., Longworth J. W., Borisov A. B., and Rhodes C. K. 1996 Biomedical three-dimensional holographic microimaging at visible, ultraviolet and X-ray wavelengths Nature Medicine 2, 939

[14] Tillman C., Persson A., Wahlstrom C. G., Svanberg S., and Herrlin K. 1995 Imaging using hard x-rays from a laser-produced plasma Appl. Phys. B 61, 333; Tillman C., Mercer I., Svanberg S., and Herrlin K. 1996 Elemental biological imaging by differential absorption with a laser-produced x-ray source $J$. Opt. Soc Am. B 13, 209; Gratz M., Tillman C., Mercer I., and Svanberg S. 1996 X-ray generation for medical applications from a laserproduced plasma Appl. Sur. Sci. 96-8, 443

[15] Jiang Z., Ikhlef A., Kieffer J.-C., Ráksi F., and Wilson K. R. 1996 Chemical reaction observed by ultrafast $\mathrm{x}$-ray absorption Springer Series in Chemical Physics 62 - Ultrafast Pheomena $X$, p.274, eds. P. F. Barabara, J. G. Fujimoto, W. H. Knox, and W. Zinth (Springer-Verlag, Berlin)

[16] Rose-Petruck C., Jimenez R., Guo T., Cavalleri A., Siders C.W., Ráksi F., Squier J.A., Walker B.C., Wilson K.R., and Barty C.P.J. 1999 Picosecond milli-angstrom lattice dynamics measured by ultrafast x-ray diffraction Nature 398310

[17] Yang J., Washio M., Endo A., and Hori T. 1999 Evaluation of femtosecond x-rays produced by Thomson scattering under linear and nonlinear interactions between a lowemittance electron beam and an intense polarized laser light Nucl. Inst. Meth. A 428556

[18] McPherson A., Thompson B. D., Borisov A. B., Boyer K., Rhodes C. K. 1994 Multiphoton-induced $\mathrm{x}$-ray emission at $4-5 \mathrm{keV}$ from $\mathrm{Xe}$ atoms with multiple core vacancies Nature 370, 631 
[19] See for example, Protopapas M., Keitel C. H. ,and Knight P. L. 1997 Atomic physics with super-high intensity lasers Rep. Prog. Phys. 60, 389

[20] Portella M.T., Bigot J.-Y., Schoenlein R.W., Cinningham J.E., and Shanck C.V. $1992 k$ space carrier dynamics in GaAs Appl. Phys. Lett. 602123

[21] Becker P.C., Fragnito H.L., Brito Cruz C.H., Fork R.L., Cunningham J.E., Henry J.E., and Shank C.V. 1988 Femtosecond photon echoes from band-to-band transitions in GaAs Phys. Rev. Lett. 611647

[22] Borisov A. B., McPherson A., Boyer K., and Rhodes C. K. 1996 Intensity dependence of the multiphoton-induced $\mathrm{Xe}(\mathrm{L})$ spectrum produced by subpicosecond $248 \mathrm{~nm}$ excitation of Xe clusters J. Phys. B: At. Mol. Opt. Phys. 29, L43

[23] Borisov A. B., McPherson A., Boyer K., and Rhodes C. K. 1996 Z- $\lambda$ imaging of $\mathrm{Xe}(\mathrm{M})$ and $\mathrm{Xe}(\mathrm{L})$ emissions from channelled propagation of intense femtosecond $248 \mathrm{~nm}$ pulses in a Xe cluster target J. Phys. B: At. Mol. Opt. Phys. 29, L113

[24] Kondo K, Borisov A. B., Jordan C., Boyer K., McPherson A., Schroeder W. A., and Rhodes C. K. 1997 Wavelength dependence of multiphoton-induced $\mathrm{Xe}(\mathrm{M})$ and $\mathrm{Xe}(\mathrm{L})$ emissions from Xe clusters J. Phys. B: At. Mol. Opt. Phys. 30, 2707

[25] Schroeder W.A., Omenetto F.G., Borisov A.B., Longworth J.W., McPherson A., Jordan C., Boyer K., Kondo K, and Rhodes C. K. 1998 Pump laser wavelength-dependent control of the efficiency of kilovolt x-ray emission from atomic clusters J. Phys. B: At. Mol. Opt. Phys. 31, 5031

[26] Bouma B., Luk T. S., Boyer K., and Rhodes C. K. 1993 High-brightness subpicosecond terawatt $\mathrm{KrF}^{*}$ system driven with a frequency converted self-mode-locked pulsecompressed Ti: $\mathrm{Al}_{2} \mathrm{O}_{3}$ laser J. Opt. Soc. Am. B 10, 1180

[27] McPherson A., Luk T. S., Thompson B. D., Borisov A. B., Shiryaev O. B., Chen X., Boyer K., and Rhodes C. K. 1994 Multiphoton induced x-ray emission from $\mathrm{Kr}$ clusters on M-shell $(\sim 100 \AA)$ and L-shell $(\sim 6 \AA)$ transitions Phys. Rev. Lett. 72, 1810

[28] Boyer K., Thompson B. D., McPherson A., and Rhodes C. K. 1994 Evidence for coherent motions in multiphoton $\mathrm{x}$-ray production from $\mathrm{Kr}$ and Xe clusters J. Phys. B: At. Mol. Opt. Phys. 27, 4373

[29] Borisov A.B., McPherson A., Thompson B.D., Boyer K., and Rhodes C.K. 1995 Ultrahigh power compression for $\mathrm{x}$-ray amplification: multiphoton cluster excitation combined with non-linear channelled propagation J. Phys. B: At. Mol. Opt. Phys. 282143

[30] Cowan R. D. 1982 The Theory of Atomic Structure and Spectra (Berkley, CA: University of California Press)

[31] Powell C. J. 1976 Cross sections for ionization of inner-shell electrons by electrons Rev. Mod. Phys. 48, 33 
[32] McGuire E. J. 1977 Scaled electron cross sections in the Born approximation Phys. Rev. A 16,73

[33] Boyer K. and Rhodes C. K. 1985 Atomic inner-shell excitation induced by coherent motion of outer-shell electrons Phys. Rev. Lett. 54, 1490

[34] Boyer K. and Rhodes C. K. 1994 Superstrong coherent multi-electron intense-field interaction J. Phys. B: At. Mol. Opt. Phys. 27, L633

[35] See for example, Merzbacher E. 1970 Quantum Mechanics $2^{\text {nd }}$ edition (John Wiley \& Sons, New York)

[36] Brabec T., Ivanov M. Y., and Corkum P. B. 1996 Coulomb focusing in intense field atomic processes Phys. Rev. A 54, R2551

[37] Bethe H. 1930 Zur Theorie des Durchgangs schneller Korpuskularstrahlen durch Materie Ann. Phys. 5, 325

[38] Briggs J.S. and Taulbjerg K. 1978 Theory of inelastic atom-atom collisions in Structure and collisions of ions and atoms Ed. Sellin I.A. Topics in Current Physics no. 5 (SpringerVerlag, New York) 105

[39] Clark M.W., Schneider D., Dewitt D., McDonald J.W., Bruch R., Safronova U.I., Tolstikhina I.Yu., and Schuch R. $1993 \mathrm{Xe} L$ and $M$ x-ray emission following $\mathrm{Xe}^{44-48+}$ ion impact on Cu surfaces Phys. Rev. A 473983

[40] Borisov A.B., Shi X.M., Karpov V.B., Korobkin V.V., Shiryaev O.B., Solem J.C., McPherson A., Boyer K., and Rhodes C.K. 1994 Stable self-channeling of intense ultraviolet pulses in underdense plasma, producing channels exceeding 100 Rayleigh lengths J. Opt. Soc. Am. B 111941

[41] Borisov A.B., McPherson A., Thompson B.D., Boyer K., and Rhodes C.K. 1996 Multielectron motions induced in clusters and molecules in self-trapped channels Multiphoton Processes 1996, Proceedings of the $7^{\text {th }}$ International Conference on Multiphoton Processes, eds. P. Lambropoulos and H. Walther (Inst. Phys. Conf. Ser. No. 154, IOP Publishing, Bristol and Philadelphia 1997) p.1.

[42] Rose-Petruck C., Schafer K. J., and Barty C. P. J. 1995 Possible ionization "ignition" in laser driven plasmas Applications of Laser Plasma Radiation II (SPIE 2532) eds. M. C. Richardson and G. A. Kyrala (Bellingham: SPIE), p.272; Rose-Petruck C., Schafer K. J., Wilson K. R., and Barty C. P. J. 1997 Ultrafast electron dynamics and inner shell ionization in laser driven plasmas Phys. Rev. A 55, 1182; Rose-Petruck C., Schafer K. J., Wilson K. R., and Barty C. P. J. 1997 Simulation of ionization ignition and inner atom shell ionization in ultrafast laser driven clusters Proc. Application of High Field and Short Wovelength Sources (Santa Fe, NM). 
[43] Saradrik E. S. and Schappert G. T. 1970 Classical theory of the scattering of intense laser radiation by free electrons Phys. Rev. D 1, 2738

[44] Volkov D.M. (1935(34?)) Über eine klasse von lösungen der Diracschen gleichung Z. Phys. 94250

[45] See, for example, Wang H., Backus S., Chang Z., Wagner R., Kim K., Wang X., Umstadter D., Lei T., Murnane M., and Kapteyn 1999 Generation of 10-W average-power, 40-TW peak-power, 24-fs pulses from a Ti:sapphire amplifier system J. Opt. Soc. Am. B 16, 1790; Barty C. P. J., Guo T., Le Blanc C, Raski F., Rose-Petruck C, Squier J., Wilson K. R., Yakovlev V. V.,and Yamakawa K. 1996 Generation of 18-fs, multiterawatt pulses by regenerative pulse shaping and chirped-pulse amplification Opt. Lett. 21, 668; Chambert J. P., C. Le Blanc C., Chériaux G., Curley P., Darpentigny G., Rousseau P., Hamoniaux G., Antonetti A., and Salin F. 1996 Generation of 25-TW, 32-fs pulses at $10 \mathrm{~Hz}$ Opt. Lett. 21, 1921; Sullivan A., Bonlie J., Price D. F., and White W. E. 1996 1.1-J, 120-fs laser system based on Nd-glass-pumped Ti:sapphire Opt. Lett. 21, 603; Beaud P. A.,Richardson M., and Miesak E. J. 1995 Multi-terawatt femtosecond Cr:LiSAF laser IEEE J. Quantum Electron. QE-31, 317; Ditmire T., Nguyen H., and Perry M. D 1994 Design and performance of a multiterawatt Cr:LiSrAlF 6 laser system J. Opt. Soc. Am. B 11, 580

[46] Data in Science and Technology, Ed. in Chief R. Poerschke, Semiconductors: Group IV Elements and III-V Compounds, Ed. O. Madelung (Springer-Verlag, Berlin, 1991)

[47] Jackson, J. D. 1975 Classical Electrodynamics 2nd edition (John Wiley \& Sons, New York)

[48] Kanter E.P., Dunford R.W., Krässig, Southworth S.H. 1999 Double $K$-vacancy production in molybdenum by $\mathrm{x}$-ray photoionization Phys. Rev. Lett. 83, 508

[49] Herman F. and Skillman S. 1963 Atomic Structure Calculations (Prentice Hall, Englewood Cliff, NJ)

[50] Gibson G., Luk T.S., McPherson A., Boyer K., and Rhodes C.K. 1989 Observation of a new inner-orbital molecular transition at $55.8 \mathrm{~nm}$ in $\mathrm{N}_{2}{ }^{2+}$ produced by multiphoton coupling Phys. Rev. A 402378

[51] Nelson T.R., Borisov A.B., Cameron S., Longworth J.W., Schroeder W.A., Santoro J., VanTassle A.J., and Rhodes C.K. 1999 Hollow atom production by a shell-selective collisional ejection process in clusters and solids: $\mathrm{Xe}(\mathrm{L})$ and $\mathrm{Ba}(\mathrm{L}) 18^{\text {th }}$ International Conference on X-ray and Inner-Shell Processes (Chicago, IL) paper D60

[52] Borisov A. B., Borovskiy A. V., Shiryaev O. B., Korobkin V. V., Prokhorov A. M., Solem J. C., Luk T. S., Boyer K., and Rhodes C. K. 1992 Relativistic and charge-displacement self-channeling of intense ultrashort laser pulses in plasmas Phys. Rev. $A$ 45, 5830 
[53] Borisov A.B., Longworth J.W.L., Boyer K., and Rhodes C.K.R. 1998 Stable relativistic/charge-displacement channels in ultrahigh power density $\left(\approx 10^{21} \mathrm{~W} / \mathrm{cm}^{2}\right)$ plasmas Proc. Natl. Acad. Sci. 95, 7854

[54] Raoult F., Boscheron A.C.L., Husson D., Rouyer C., Sauteret C., and Migus A. 1999 Ultrashort intense ultraviolet pulse generation by efficient frequency tripling and adapted phase matching, Opt. Lett. 25, 354 


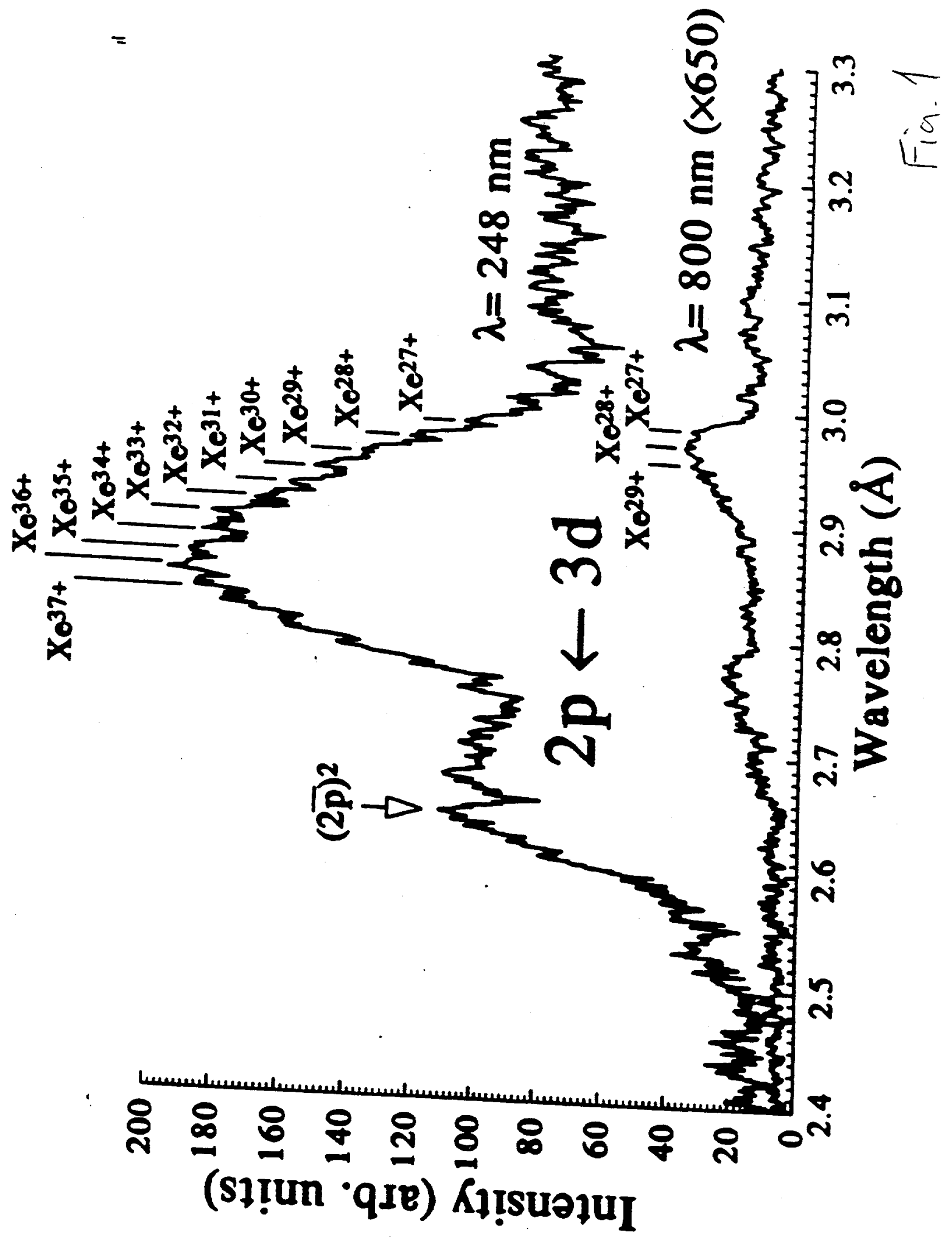




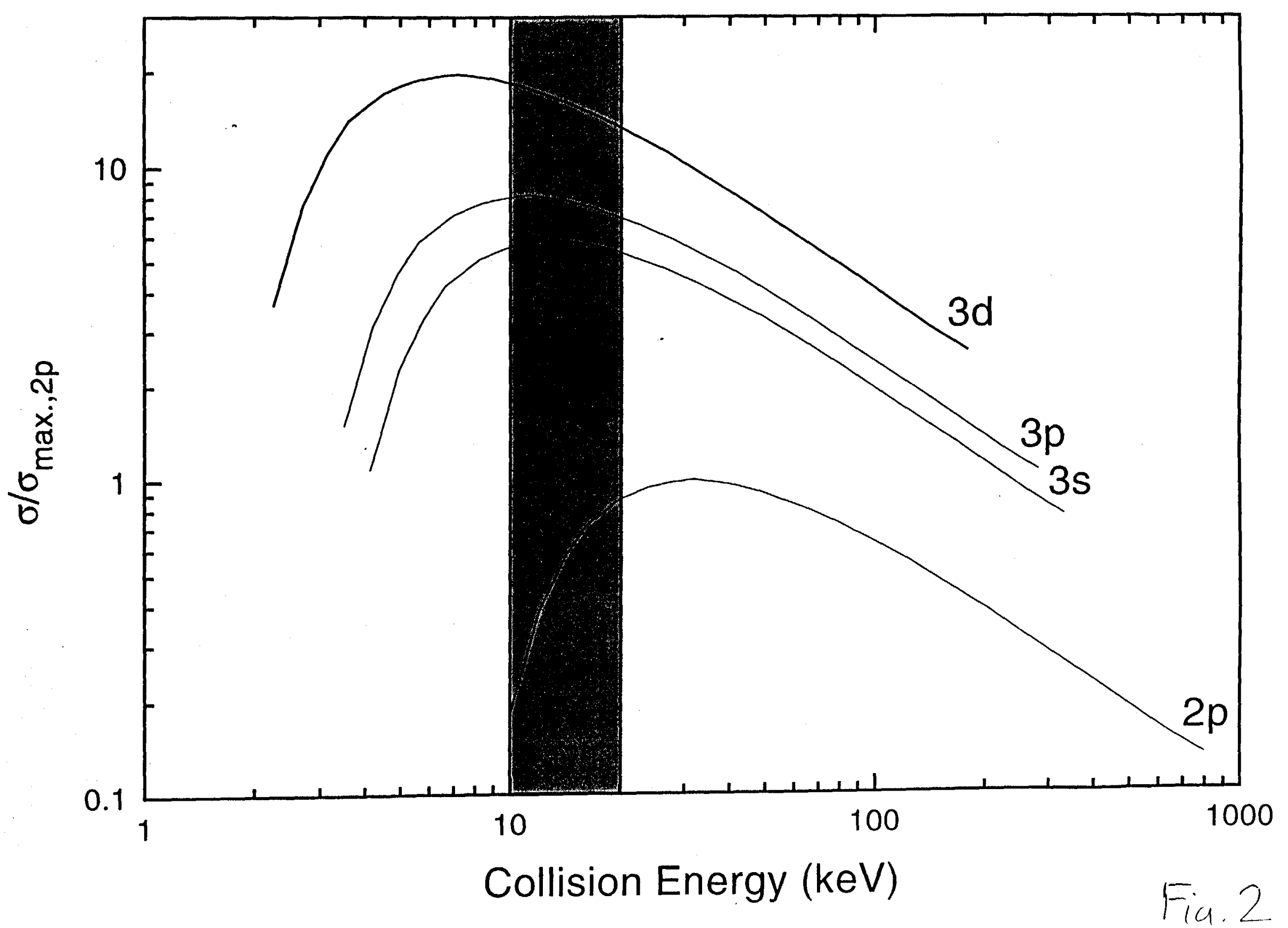




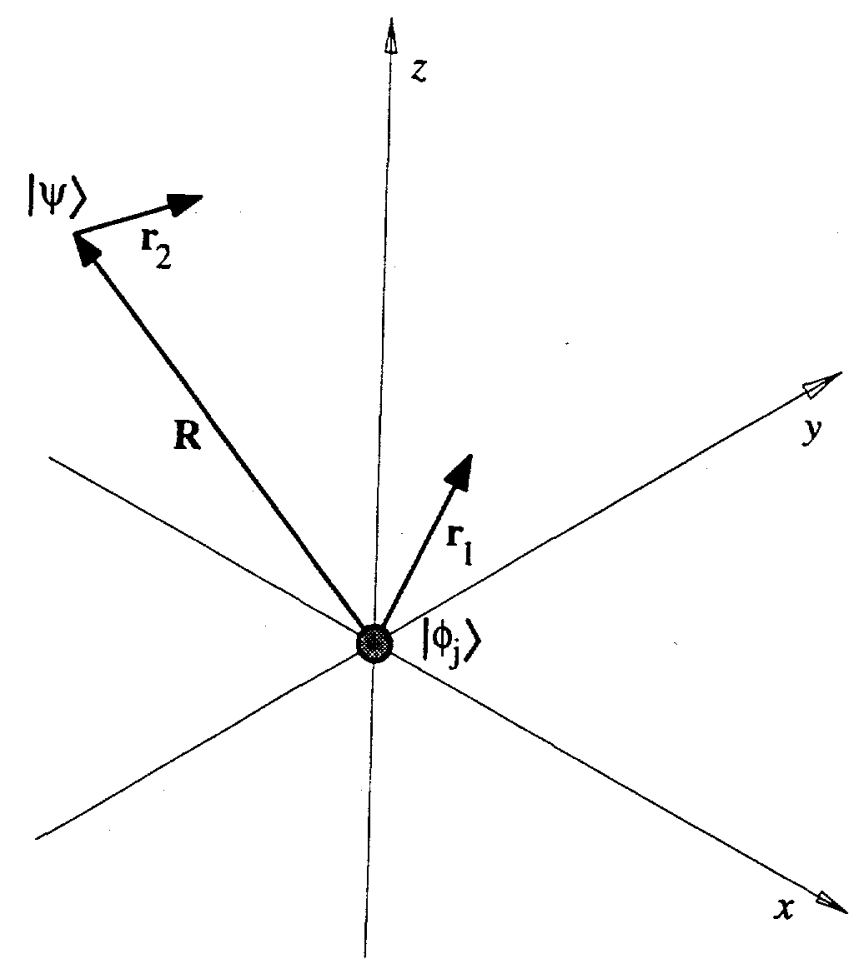

Figure 3 
hydroxenon4p_hdf
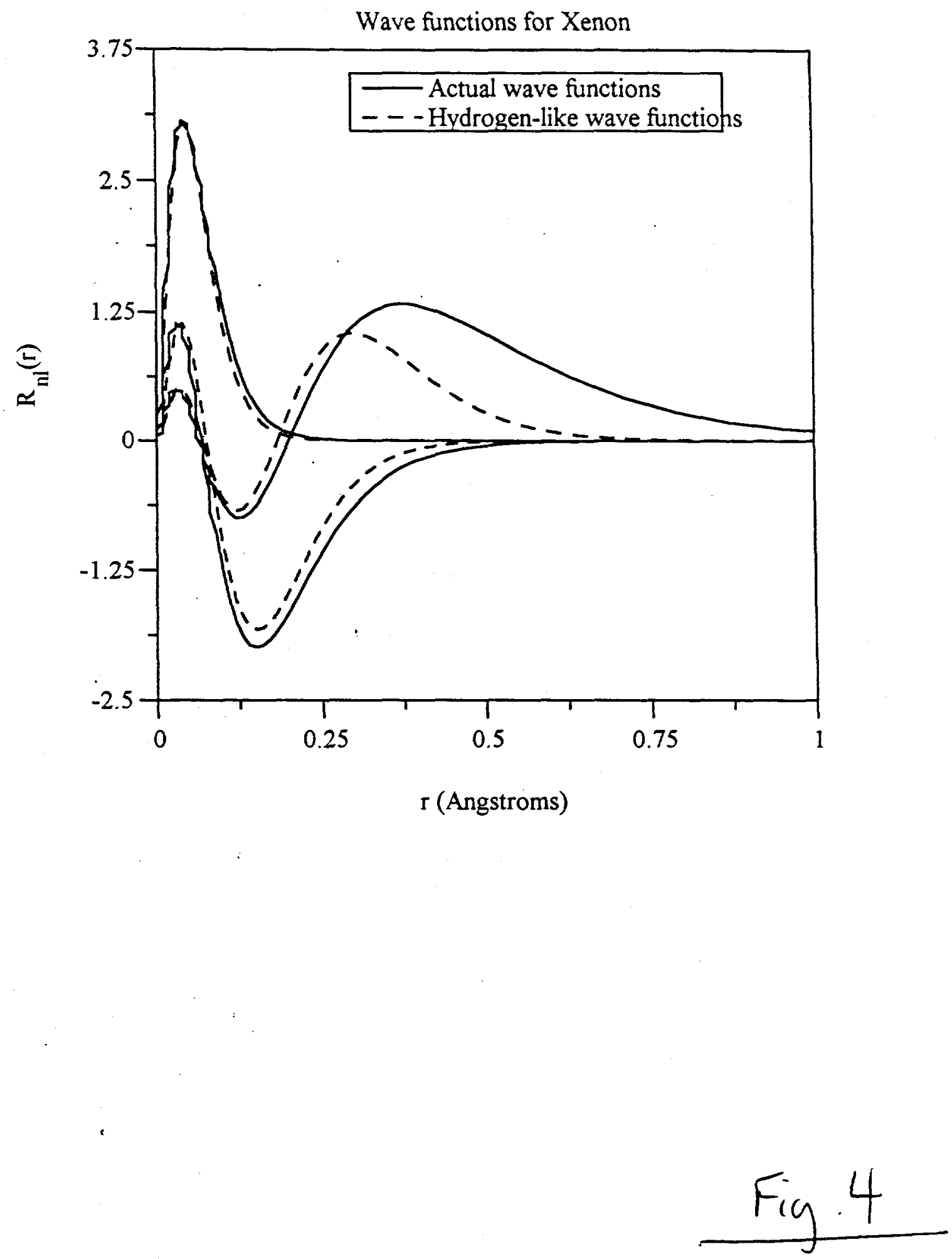


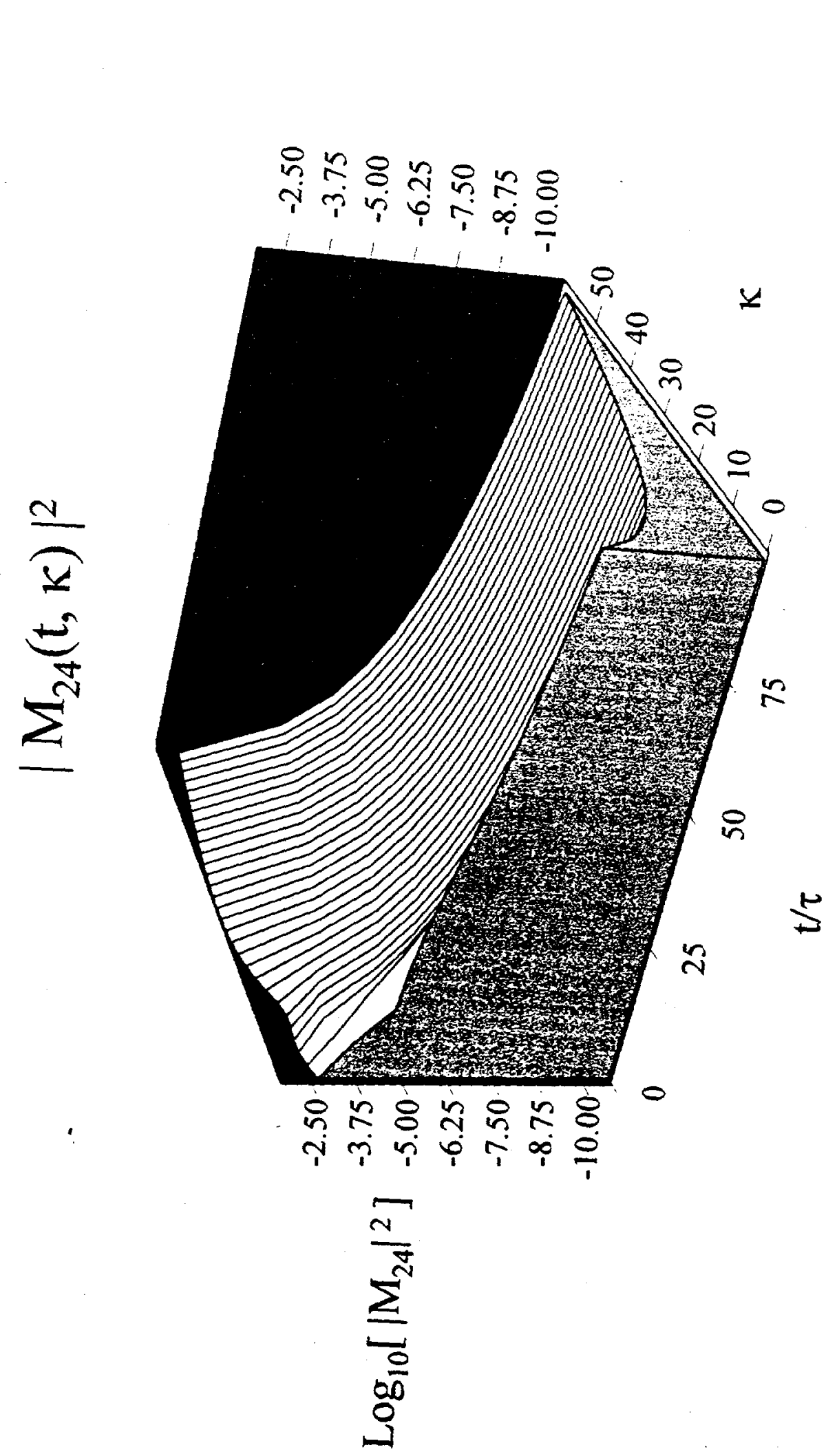




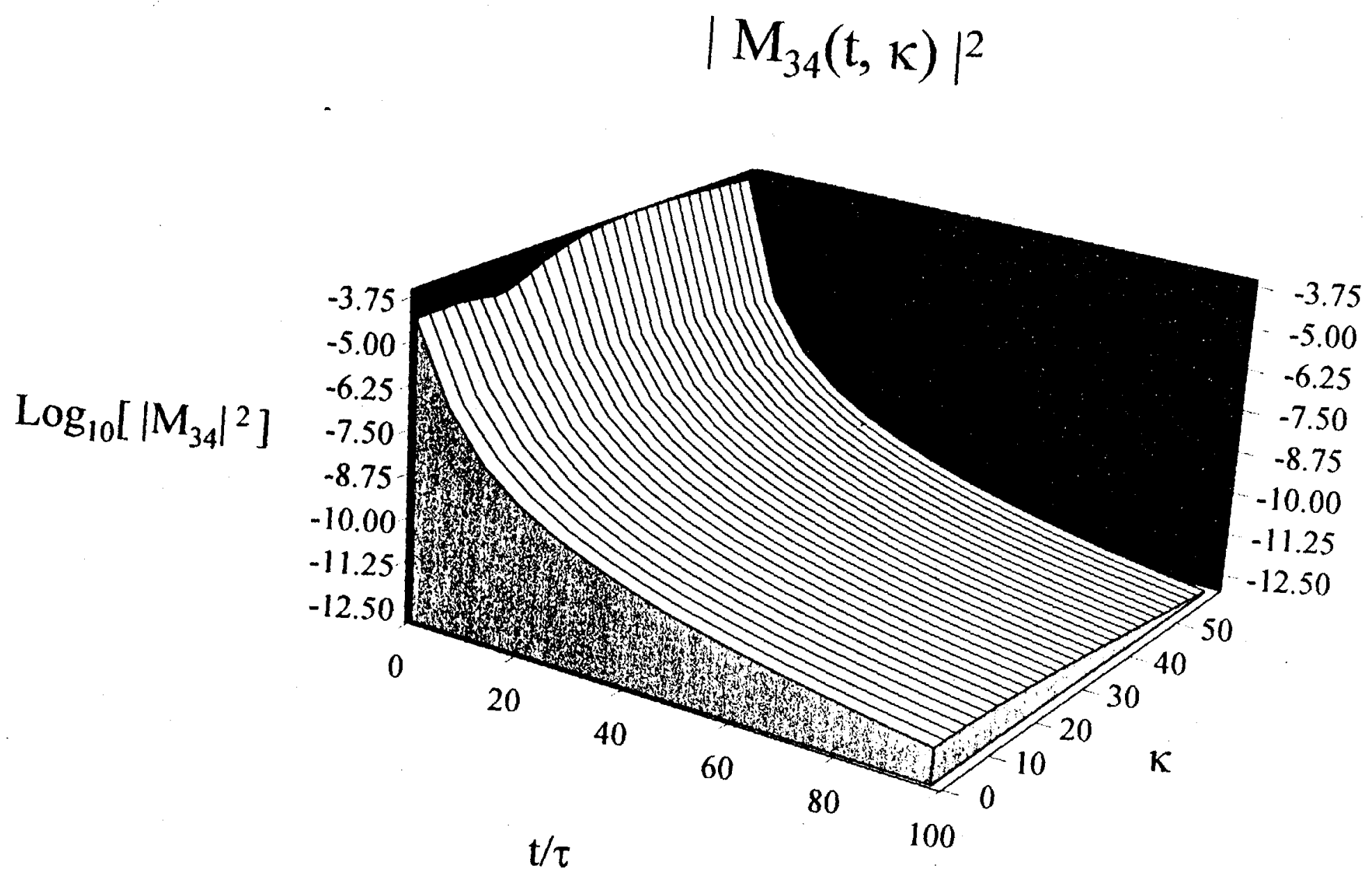




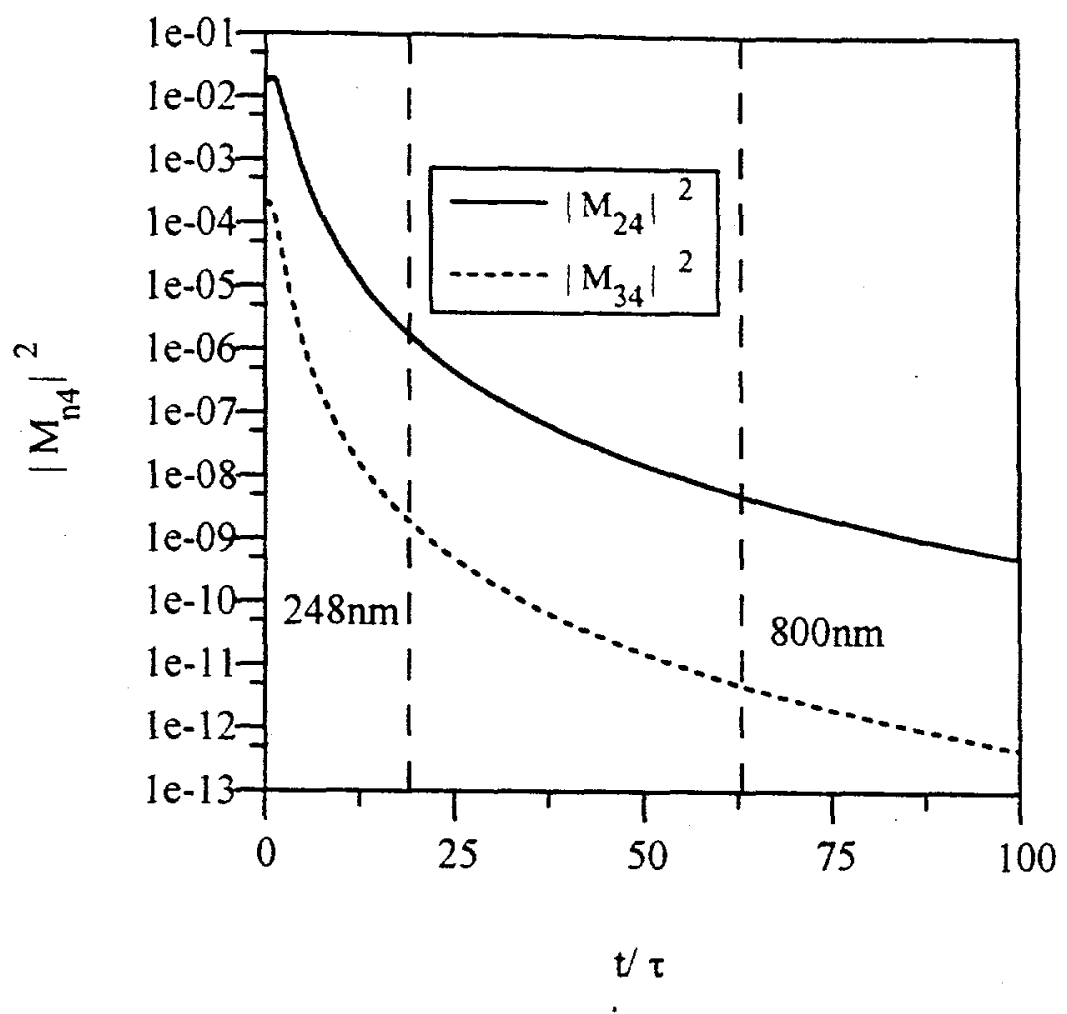

Aug 021999

Fig. 6(a)

Page 1 of 1 

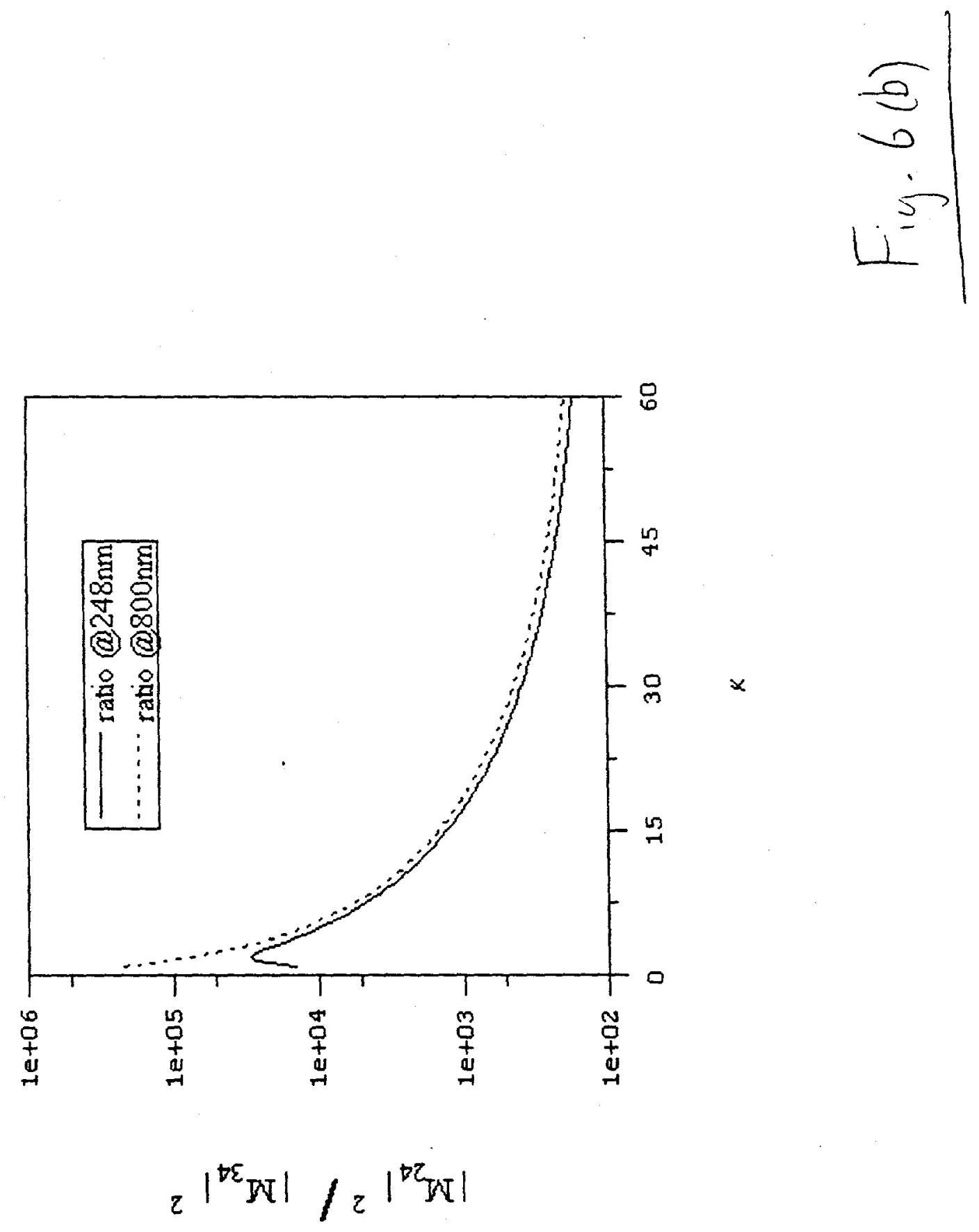

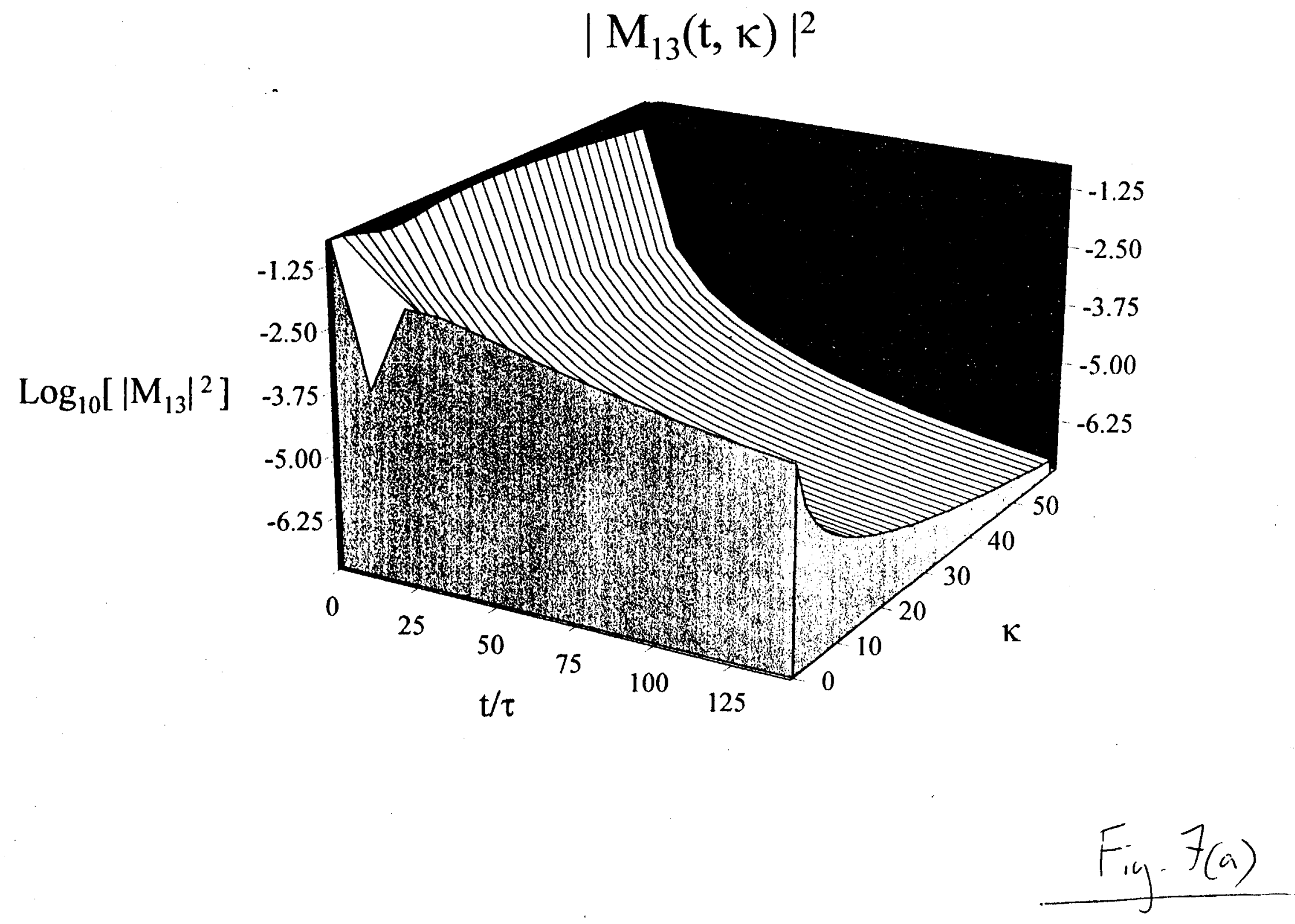

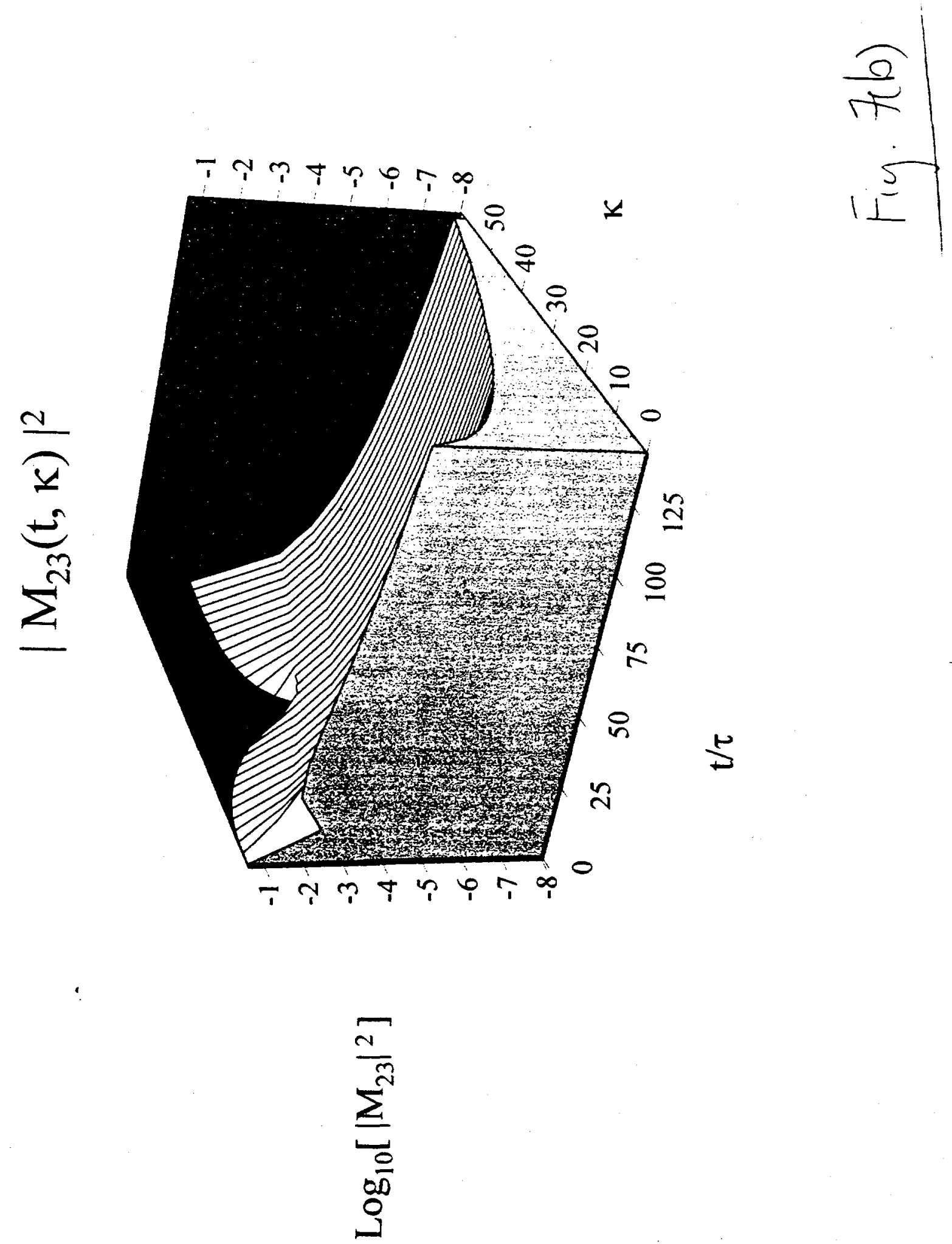

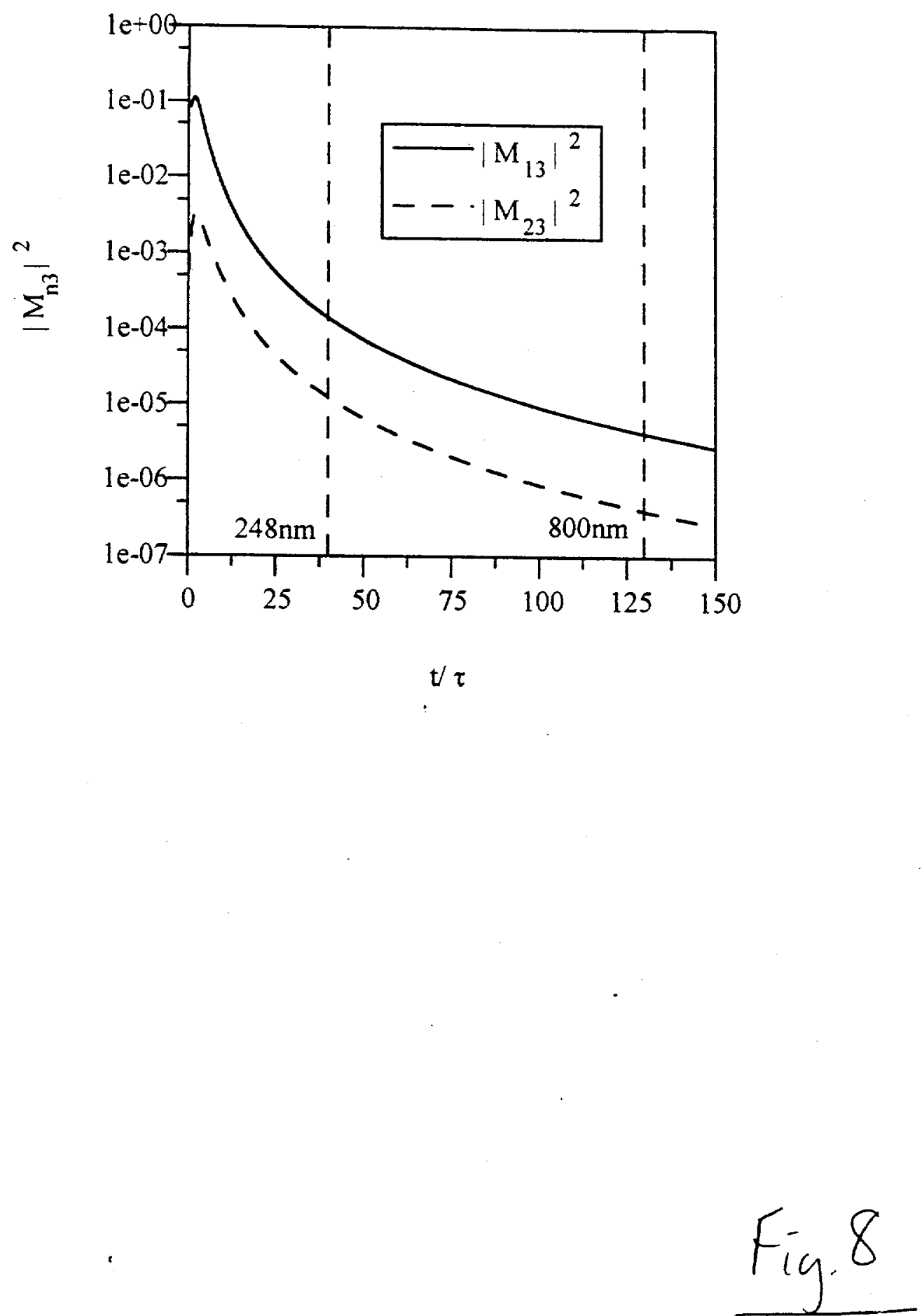